\title{
An Easy Synthetic Approach to Pyridoporphyrins by Domino Reactions
}

\author{
Cristina M. A. Alonso, ${ }^{\dagger}$ Vanda I. V. Serra ${ }^{\dagger}$ Maria G. P. M. S. Neves, ${ }^{\dagger}$ Augusto C. \\ Tomé, ${ }^{\dagger}$ Artur M. S. Silva, ${ }^{\dagger}$ Filipe A. Almeida Paz, ${ }^{\ddagger}$ José A. S. Cavaleiro $^{\dagger *}$ \\ ${ }^{\dagger}$ Department of Chemistry, QOPNA, University of Aveiro, 3810-193 Aveiro, Portugal \\ * Department of Chemistry, CICECO, University of Aveiro, 3810-193 Aveiro, Portugal
}

\section{Supporting Information}

\section{Table of contents:}

Materials \& Methods

Experimental Procedures and Spectroscopic Data for 2a and 3a

Experimental Procedures and Spectroscopic Data for $\mathbf{4}$ and $\mathbf{5}$. S4-S7

Experimental Procedures and Spectroscopic Data for 6. .S7

Experimental Procedures and Spectroscopic Data for 7. S8

Single-crystal X-ray Diffraction Studies of 7 S8-S10

${ }^{1} \mathrm{H}$ NMR Spectrum of compound 2a (Figure S1).

${ }^{13} \mathrm{C}$ NMR Spectrum of compound 2a (Figure S2)

COSY Spectra of compound 2a (Figures S3 and S4)

HSQC Spectra of compound 2a (Figures S5-S8).

Partial NOESY Spectrum of compound 2a (Figure S9).

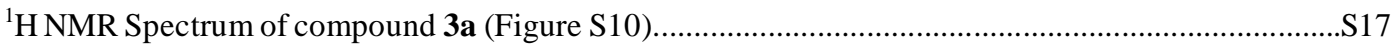

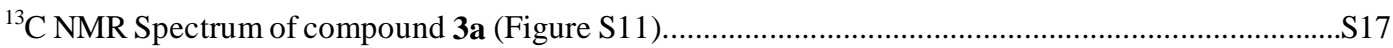

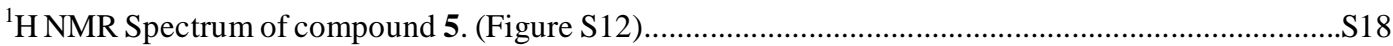

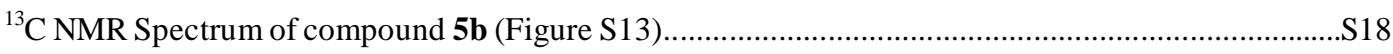

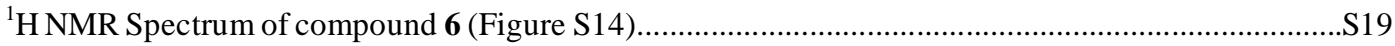

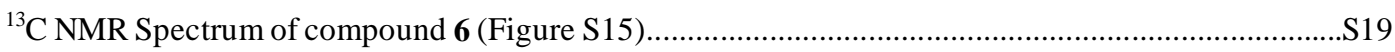

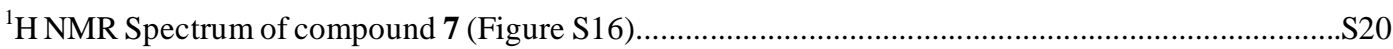

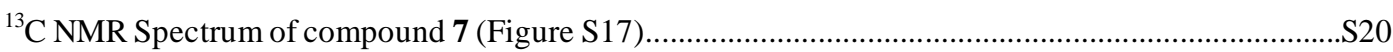

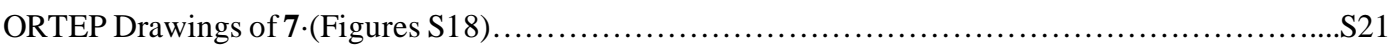

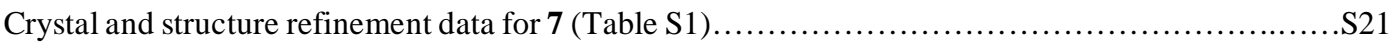

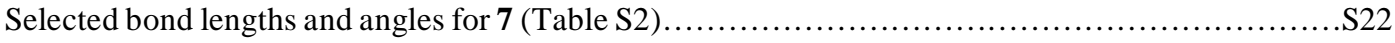

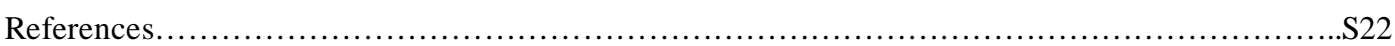




\section{Material \& Methods}

${ }^{1} \mathrm{H}$ and ${ }^{13} \mathrm{C}$ NMR spectra were recorded on Bruker Avance 500 (at 500.13 and $125.77 \mathrm{MHz}$, respectively) and AMX 300 (at 300.13 and $75.47 \mathrm{MHz}$, respectively) spectrometers. $\mathrm{CDCl}_{3}$ was used as solvent and TMS as internal reference, the chemical shifts are expressed in $\delta(\mathrm{ppm})$ and the coupling constants $(J)$ in Hertz $[\mathrm{Hz}]$. Unequivocal ${ }^{1} \mathrm{H}$ assignments were made using 2D COSY and NOESY experiments (mixing time of $800 \mathrm{~ms}$ ), while ${ }^{13} \mathrm{C}$ assignments were made on the basis of 2D HSQC and $\mathrm{HMBC}$ experiments (delay for long-range $J \mathrm{C} / \mathrm{H}$ couplings were optimized for $7 \mathrm{~Hz}$ ). Mass spectra and HRMS were recorded on VG AutoSpec Q and $\mathrm{M}$ mass spectrometers using $\mathrm{CHCl}_{3}$ as solvent and 3nitrobenzyl alcohol (NBA) as matrix. The UV-Vis spectra were recorded on a Uvikon spectrophotometer using $\mathrm{CHCl}_{3}$ as solvent. Melting points were measured on a Reichert Thermovar apparatus fitted with a microscope and are uncorrected. Preparative thin-layer chromatography was carried out on $20 \times 20 \mathrm{~cm}$ glass plates coated with Merck 60 silica gel ( $0.5 \mathrm{~mm}$ thick). Column chromatography was carried in silica gel (Merck, 230-400 mesh). Analytical TLC was carried out on precoated sheets with silica gel (Merck $60,0.2 \mathrm{~mm}$ thick). Toluene was distilled from benzophenone and sodium metal, pyridine was distilled from potassium hydroxide, all other solvents and reagents were used without further purification.

The aminoporphyrins 1a-d were prepared by nitration of the corresponding $\beta$-unsubstituted porphyrins, followed by reduction of the nitro group, acordding to literature procedures. ${ }^{1,2}$

\section{Experimental Procedures and Spectroscopic Data for 2a and 3a}

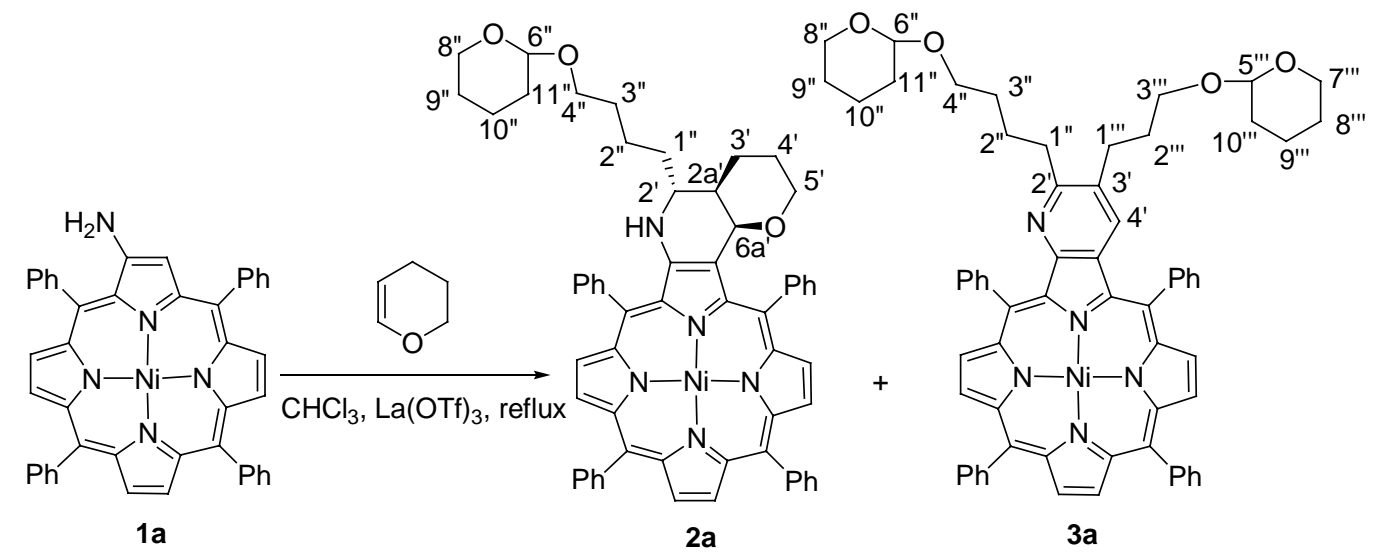

3,4-Dihydro-2 $\mathrm{H}$-pyran $(0.05 \mathrm{~mL}, 0.548 \mathrm{mmol})$ and lanthanum triflate $(2 \mathrm{mg}, 3.41 \mu \mathrm{mol})$ were added to a stirred solution of 2-aminoporphyrin $1 \mathrm{a}(29.1 \mathrm{mg}, 0.059 \mathrm{mmol})$ in chloroform $(3 \mathrm{~mL})$. The reaction mixture was heated at reflux until complete consumption of the starting porphyrin (1 day, as indicated by TLC). The reaction mixture was allowed to reach room temperature, it was diluted with chloroform and then it was washed with water. The organic solution was dried $\left(\mathrm{Na}_{2} \mathrm{SO}_{4}\right)$ and concentrated under reduced pressure. The products were separated by preparative TLC using a mixture of dichloromethane:light petroleum (4:1) as eluent. 
\{1,2,2a,3,4,6a-Hexahydro-7,12,17,22-tetraphenyl-2-[4-(tetrahydro-2H-pyran-2-yloxy)butyl]-2Hpyrano[2',3':4,5]pyrido[2,3-b]porphyrinato\}nickel(II), 2a (6.3 mg, 13\%). $\left.{ }^{1} \mathrm{H} \mathrm{NMR} \mathrm{(500} \mathrm{MHz,} \mathrm{CDCl}_{3}\right)$ $\delta$ 0.96-1.15 and 1.17-1.28 (2m, 4H, $\left.\mathrm{CH}_{2}\right), 1.37-1.46\left(\mathrm{~m}, 2 \mathrm{H}, \mathrm{H}-1{ }^{\prime}{ }^{\prime}\right), 1.50-1.68\left(\mathrm{~m}, 7 \mathrm{H}, 3 \mathrm{CH}_{2}\right.$ and $1 \mathrm{H}$ of $\left.\mathrm{CH}_{2}\right), 1.71-1.80$ and 1.82-1.96 (2m, 4H, H-2a' and $3 \mathrm{H}$ of $\left.\mathrm{CH}_{2}\right), 2.83$ (t, $J=10.7 \mathrm{~Hz}, 1 \mathrm{H}, \mathrm{H}-5$ ' ), 3.38-3.44 and 3.55-3.58 (2m, 2H, H-4' ' and H-8' '), 3.75-3.83 (m, 2H, H-5' and H-4' ' or H-8' '), 3.86-3.95 (m, 2H, H-2' and H-4', or H-8', ), 3.99 (d, $J=1.1 \mathrm{~Hz}, 1 \mathrm{H}, \mathrm{H}-6 \mathrm{a}$ '), 4.33 (s, 1H, NH), 4.63-4.66 (m, 1H, H-6'), 7.54-7.71 (m, 14H, 12H-m,p-Ph and 2H-o-Ph), 7.94-8.10 (m, 6H, H-o-Ph), 8.42 (d, J = 5.0 Hz, 1H, H- $\beta$ ), $8.56(\mathrm{~d}, J=5.0 \mathrm{~Hz}, 1 \mathrm{H}, \mathrm{H}-\beta), 8.58-8.60(\mathrm{~m}, 4 \mathrm{H}, \mathrm{H}-\beta) .{ }^{13} \mathrm{C} \mathrm{NMR}\left(125 \mathrm{MHz}, \mathrm{CDCl}_{3}\right) \delta 19.7$ and 19.8, 20.77 and 20.84, 21.5, 24.6, 25.5, 29.9 and 30.0, 30.8, 32.1 (C-1'’), 36.3 (C-2a'), 48.56 and 48.60 (C-2'), 62.4 and 62.5, 67.46 and 67.48 (C-4', C-8'”), 68.3 (C-6'), 70.5 (C-6a'), 98.9 and 99.1 (C-6'”), 113.5, $114.8,115.3,117.9,120.0,126.2 ; 126.80,126.83,127.4,127.5,127.6,128.4$ and $128.6(\mathrm{C}-m, p-\mathrm{Ph})$; $129.4(\mathrm{C}-\beta), 130.4 ; 130.8,131.2,131.4,132.1$ and $132.4(\mathrm{C}-\beta) ; 132.0,132.6,133.5$ and $133.6(\mathrm{C}-o-\mathrm{Ph})$; $139.8,140.1,140.3,140.77,140.82,141.2,141.5,142.3,142.7,144.2,153.1(\mathrm{C}-2)$. UV-vis $\left(\mathrm{CHCl}_{3}\right) \lambda_{\max }$ ( $\log \varepsilon) 413$ (5.21), 534 (3.95), 582 (3.91). HRMS (FAB) calcd for $\mathrm{C}_{59} \mathrm{H}_{53} \mathrm{~N}_{5} \mathrm{O}_{3} \mathrm{Ni}(\mathrm{M})^{\bullet+} 937.3481$, found 937.3502. Anal. Calcd for $\mathrm{C}_{59} \mathrm{H}_{53} \mathrm{~N}_{5} \mathrm{O}_{3} \mathrm{Ni} \cdot 1 / 2 \mathrm{H}_{2} \mathrm{O}: \mathrm{C}, 74.77 ; \mathrm{H}, 5.74 ; \mathrm{N}$ 7.39. Found: C, 74.88; H, 5.57; N, 7.23 .

\{5,10,15,20-Tetraphenyl-2-[4-(tetrahydro-2H-pyran-2-yloxy)butyl]-3-[3-(tetrahydro-2H-pyran-2yloxy)propyl]-pyrido[2,3-b]porphyrinato\}nickel(II), 3a (19.8 mg, 38\%). $\left.{ }^{1} \mathrm{H} \mathrm{NMR} \mathrm{(300} \mathrm{MHz,} \mathrm{CDCl}_{3}\right)$ $\delta$ 1.50-1.64 (m, 12H, CH$), 1.72-1.92\left(\mathrm{~m}, 6 \mathrm{H}, \mathrm{CH}_{2}\right), 2.67-2.79(\mathrm{~m}, 4 \mathrm{H}, \mathrm{H}-1$ ', and H-1', '), 3.36-3.49 (m,

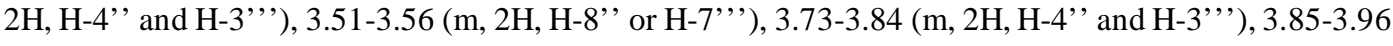
(m, 2H, H-8', or H-7' ', '), 4.59-4.61 and 4.63-4.65 (2m, 2H, H-6' and H-5','), 6.90 (s, 1H, H-4'), 7.587.80 (m, 12H, H-m,p-Ph), 7.83-7.86 (m, 2H, H-o-Ph), 7.91-8.01 (m, 6H, H-o-Ph), 8.62-8.72 (m, 6H, Hß). ${ }^{13} \mathrm{C} \mathrm{NMR}\left(75 \mathrm{MHz}, \mathrm{CDCl}_{3}\right) \delta$ 19.7, 19.8, 24.6, 25.5, 29.3 (C-1','), 29.8, 29.9, 30.8, 30.9, 35.0 (C-1' '), 62.37 and 62.42 (C-8', C-7','), 66.8 (C-3','), 67.8 (C-4'”), 98.8 and 98.9 (C-6', C-5','), 115.4, 115.7, 119.97, 120.01; 126.8, 126.9, 127.0, 127.8, 127.9 and 128.4 (C-m,p-Ph); 130.6 (C-4'); 130.7, 131.42, 131.44, 131.6, 131.7, 131.8, 132.45 and 132.52 (C- $\beta$ ); 132.8, 132.9 and $133.5(\mathrm{C}-o$-Ph), 135.5, 136.2, 140.5, 140.6, 140.7, 140.8, 141.3, 141.6, 141.7, 143.2, 144.4, 155.2 (C-2), 159.0 (C-2'). UV-vis $\left(\mathrm{CHCl}_{3}\right)$ $\lambda_{\max }(\log \varepsilon) 423$ (5.38), 538 (4.19), 577 (3.99). HRMS (FAB) calcd for $\mathrm{C}_{64} \mathrm{H}_{59} \mathrm{~N}_{5} \mathrm{O}_{4} \mathrm{Ni}(\mathrm{M})^{*+} 1019.3921$, found 1019.3938. Anal. Calcd for $\mathrm{C}_{64} \mathrm{H}_{59} \mathrm{~N}_{5} \mathrm{O}_{4} \mathrm{Ni}: \mathrm{C}, 75.30 ; \mathrm{H}, 5.83 ; \mathrm{N}, 6.86$. Found: C, 75.13; H, 5.85; N, 6.90 . 
Experimental Procedures and Spectroscopic Data for 4 and 5

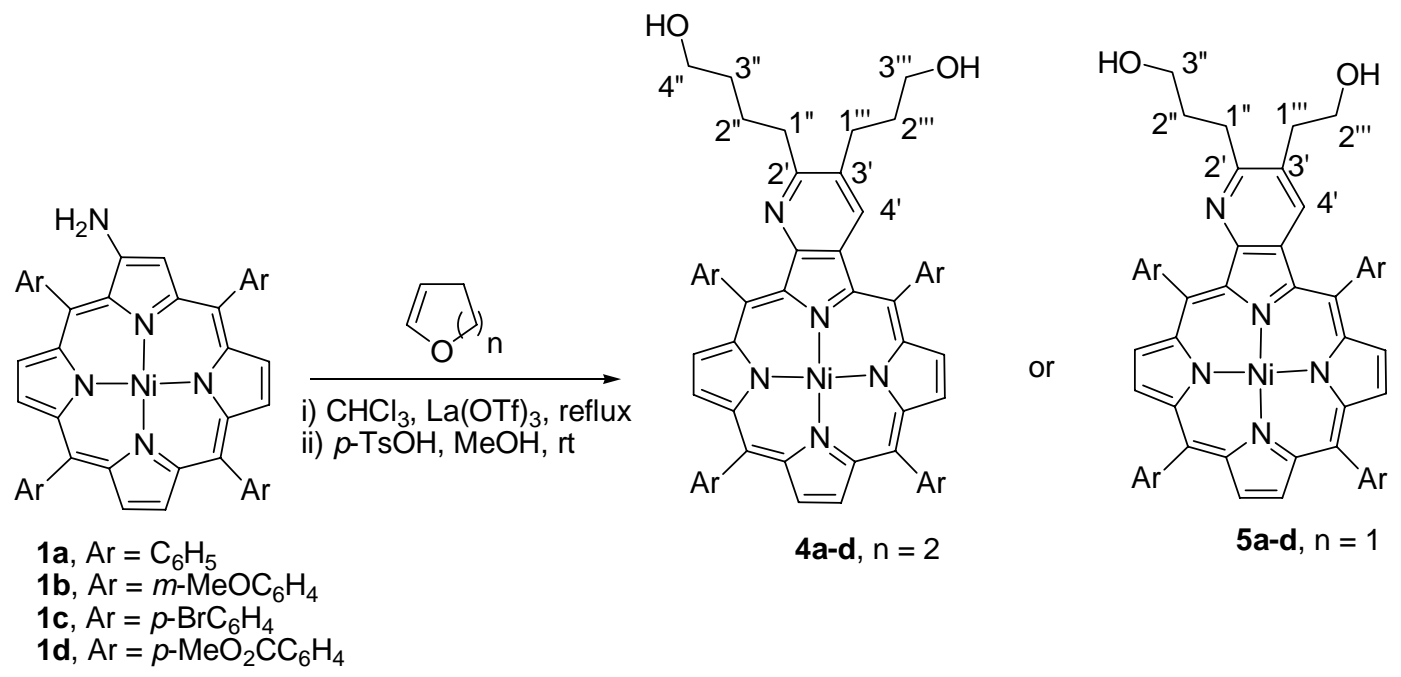

3,4-Dihydro- $2 \mathrm{H}$-pyran $(0.05 \mathrm{~mL}, 0.548 \mathrm{mmol})$ or 2,3-dihydrofuran $(0.05 \mathrm{~mL}, 0.661 \mathrm{mmol})$ and lanthanum triflate $(2 \mathrm{mg}, 3.41 \mu \mathrm{mol})$ were added to a stirred solution of 2-aminoporphyrin 1a-1d $(0.038$ mmol) in chloroform. The reaction mixture was heated at reflux until the TLC confirmed complete consumption of the starting porphyrin (1 day for $\mathbf{4 a}, \mathbf{5 a}, \mathbf{5 c}, \mathbf{5 d}$ and 4 days for $\mathbf{4 b}, \mathbf{4 c}, \mathbf{4 d}, \mathbf{5 b}$ ). The reaction mixture was allowed to reach room temperature and $p$-toluenesulfonic acid $(60 \mathrm{mg})$ in methanol $(2 \mathrm{~mL})$ was added. The mixture was stirred for 4 hours, it was diluted with chloroform and then washed successively with a saturated aqueous solution of $\mathrm{NaHCO}_{3}$ and with water. The organic layer was dried $\left(\mathrm{Na}_{2} \mathrm{SO}_{4}\right)$ and concentrated under reduced pressure. The products were separated by column chromatography using dichloromethane as the eluent and were crystallized from dichloromethane: $n$ hexane.

\section{[2-(4-Hydroxybutyl)-3-(3-hydroxypropyl)-5,10,15,20-tetraphenylpyrido[2,3-}

b]porphyrinato]nickel(II), 4a (22.3 mg, 69\%). ${ }^{1} \mathrm{H}$ NMR (300 MHz, CDCl $) \delta 1.51-1.59$ (m, 4H, H-2",

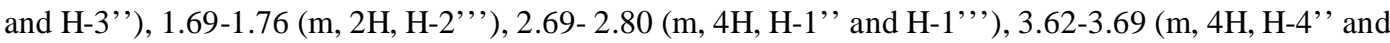
H-3" '), 6.92 (s, 1H, H-4'), 7.61-7.82 (m, 12H, H-m,p-Ph), 7.84-7.88 (m, 2H, H-o-Ph), 7.92-8.00 (m, 6H, H-o-Ph), 8.62-8.70 (m, 6H, H- $\beta$ ). ${ }^{13} \mathrm{C}$ NMR (75 MHz, CDCl 3 ) $\delta 23.5$ (C-2'”), 28.6 (C-1 '"'), 32.4 (C-2'”'), 32.8 (C-3"), 34.7 (C-1'”), 61.9 (C-3"'), 62.8 (C-4"), 115.4, 115.7, 120.0, 120.1; 126.8, 126.9, 127.8, 127.9 and 128.3 (C-m,p-Ph); 130.7 (C-4', C- $\beta$ ); 131.39, 131.49, 131.52 and 131.6 (C- $\left.\beta, C^{\prime}-3^{\prime}\right) ; 132.5$ and 132.6 (C- $\beta$ ); 132.9, 133.0 and 133.5 (C-o-Ph); 135.4, 136.0, 140.5, 140.7, 140.8, 140.9, 141.4, 141.68, 141.72, 143.1, 144.5, 155.2 (C-2), 158.7 (C-2). UV-vis $\left(\mathrm{CHCl}_{3}\right) \lambda_{\max }(\log \varepsilon) 423$ (5.37), 538 (4.19), 577 (3.99). HRMS (FAB) calcd for $\mathrm{C}_{54} \mathrm{H}_{43} \mathrm{~N}_{5} \mathrm{O}_{2} \mathrm{Ni}(\mathrm{M})^{\circ+}$ 851.2730, found 851.2770. Anal. Calcd for $\mathrm{C}_{54} \mathrm{H}_{43} \mathrm{~N}_{5} \mathrm{O}_{2} \mathrm{Ni}: \mathrm{C}, 76.07 ; \mathrm{H}, 5.08 ; \mathrm{N}, 8.21$ Found: C, 76.46; H, 4.97; N, 8.16.

[2-(4-Hydroxybutyl)-3-(3-hydroxypropyl)-5,10,15,20-tetrakis(3-methoxyphenyl)pyrido[2,3-

b]porphyrinato]nickel(II), 4b (17.0 mg, 46\%). ${ }^{1} \mathrm{H}$ NMR (500 MHz, $\left.\mathrm{CDCl}_{3}\right) \delta 1.58-1.63$ (m, 4H, H-2"' and H-3"'), 1.77-1.82 (m, 2H, H-2"' '), 2.75 and 2.78 (2t, $J=7.2 \mathrm{~Hz}, 2 \mathrm{H}, \mathrm{H}-1$ '"'), 2.80-2.86 (m, 2H, H- 
1', ), 3.66 (t, $J=6.1 \mathrm{~Hz}, 2 \mathrm{H}, \mathrm{H}-3$ ', '), 3.68-3.72 (m, 2H, H-4', ), 3.89 and 3.90 (2s, 6H, OCH $), 3.92$ (s, 6H, $\mathrm{OCH}_{3}$ ), 7.03 (s, 1H, H-4'), 7.20-7.25 (m, 3H, H-4-Ph-5,10,15), 7.36 (dd, $J=2.1$ and 8.2 Hz, 1H, H-4-Ph20), 7.44 (br d, $J=7.4$ Hz, 1H, H-6-Ph-20,), 7.47-7.62 (m, 10H, H-Ph), 7.66 (t, J = 7.9 Hz, 1H, H-5-Ph20), 8.71 and $8.73(2 \mathrm{AB}, J=5.3 \mathrm{~Hz}, 4 \mathrm{H}, \mathrm{H}-\beta), 8.77$ and $8.76(\mathrm{AB}, J=4.9 \mathrm{~Hz}, 2 \mathrm{H}, \mathrm{H}-\beta) .{ }^{13} \mathrm{C} \mathrm{NMR}(125$

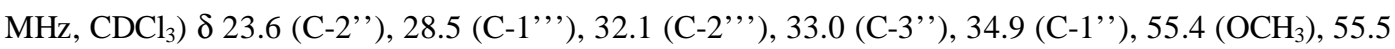
$\left(\mathrm{OCH}_{3}\right), 55.6\left(\mathrm{OCH}_{3}\right), 61.7$ (C-3','), 62.8 (C-4'’), 113.3 and 113.5 (C-4-Ph-5,10,15), 114.3 (C-4-Ph-20), 115.1, 115.3; 118.5, 118.7 and 119.4 (C-2-Ph-5,10,15); 119.7, 119.8, 126.1 (C-6-Ph-20); 125.7, 126.5, 127.6 and 127.8 (C-5-Ph, C-6-Ph); 128.9 (C-5-Ph-20), 130.76 and 130.83 (C- $\beta$, C-4'); 131.4, 131.47, $131.55,131.62,132.5$ and 132.6 (C- $\beta$, C-3); 135.2, 135.9, 140.57, 140.62, 141.5, 141.6, 141.7, 142.1, 142.7, 143.0, 144.2, 155.0 (C-2), 158.1, 158.3, 158.8 (C-2'), 159.1 (C-3-Ph-20). UV-vis $\left(\mathrm{CHCl}_{3}\right) \lambda_{\max }$ (log ع) 423 (5.32), 538 (4.15), 577 (3.99). HRMS (FAB) calcd for $\mathrm{C}_{58} \mathrm{H}_{51} \mathrm{~N}_{5} \mathrm{O}_{6} \mathrm{Ni}(\mathrm{M})^{\cdot+} 971.3193$, found 971.3157. Anal. Calcd for $\mathrm{C}_{58} \mathrm{H}_{51} \mathrm{~N}_{5} \mathrm{O}_{6} \mathrm{Ni}$ : C, 71.61; H, 5.28; N, 7.20. Found: C, 71.39; H, 5.40; N, 6.67. [2-(4-Hydroxybutyl)-3-(3-hydroxypropyl)-5,10,15,20-tetrakis(4-bromophenyl)pyrido[2,3-

b]porphyrinato]nickel(II), 4c (13.5 mg, 31\%). ${ }^{1} \mathrm{H}$ NMR (300 MHz, CDCl $)_{3} \delta 1.51-1.65$ (m, 4H, H-2', and H-3', ), 1.67-1.75 (m, 2H, H-2,', ), 2.69-2.77 (m, 4H, H-1', and H-1 ','), 3.64 (t, 4H, J = 6.1 Hz, H-4", and H-3',' ), 6.90 (s, 1H, H-4'), 7.62-7.85 (m, 16H, H-Ph), 8.57 (AB, J=4.5 Hz, 2H, H- $\beta$ ), 8.57 and 8.63 $(2 \mathrm{AB}, J=5.0 \mathrm{~Hz}, 2 \mathrm{H}, \mathrm{H}-\beta), 8.61(\mathrm{AB}, J=5.0 \mathrm{~Hz}, 2 \mathrm{H}, \mathrm{H}-\beta) .{ }^{13} \mathrm{C}$ NMR $\left(75 \mathrm{MHz}, \mathrm{CDCl}_{3}\right) \delta 24.4(\mathrm{C}-2$ '”), 28.9 (C-1','), 32.4 (C-2','), 32.7 (C-3'”), 35.0 (C-1'”), 62.0 (C-3','), 62.4 (C-4'”), 114.4, 114.6, 119.06, 119.09, 121.6, 122.7, 123.1, 130.1 (C-Ph), 130.4 (C-4' and C-Ph), 130.6 (C- $\beta$ ), 131.3 (C-o-Ph); 131.56, 131.62, 132.3, 132.67 and 132.72 (C- $\beta) ; 134.5,134.7$ and $135.0(\mathrm{C}-\mathrm{Ph}) ; 135.7,136.3,139.3,139.7$, 140.2, 140.7, 140.8, 141.6, 141.7, 143.2, 144.3, 155.0 (C-2), 159.7 (C-2'). UV-vis $\left(\mathrm{CHCl}_{3}\right) \lambda_{\max }(\log \varepsilon)$ 425 (5.33), 540 (4.13), 579 (3.96). HRMS (ESI) calcd for $\mathrm{C}_{54} \mathrm{H}_{40} \mathrm{~N}_{5} \mathrm{O}_{2} \mathrm{Br}_{4} \mathrm{Ni}(\mathrm{M}+\mathrm{H})^{+} 1167.9222$, found 1167.9212 .

[2-(4-Hydroxybutyl)-3-(3-hydroxypropyl)-5,10,15,20-tetrakis(4-carboxymethylphenyl)pyrido[2,3b]porphyrinato]nickel(II), 4d (13.1 mg, 33\%). ${ }^{1} \mathrm{H}$ NMR $\left(300 \mathrm{MHz}, \mathrm{CDCl}_{3}\right) \delta 1.49-1.54$ (m, 4H, H-2', and H-3'’), 1.66-1.73 (m, 2H, H-2','), 2.71 (t, $J=7.4$ Hz, 2H, H-1','), 2.74 (t, J=6.9 Hz, 2H, H-1' '), 3.64 (t, $J=5.4 \mathrm{~Hz}, 4 \mathrm{H}, \mathrm{H}-4$ ' ' and H-3', '), 4.08, 4.09 and $4.12\left(3 \mathrm{~s}, 12 \mathrm{H}, \mathrm{CO}_{2} \mathrm{CH}_{3}\right), 6.90$ (s, 1H, H-4'), $7.98(\mathrm{~d}, J=8.5 \mathrm{~Hz}, 2 \mathrm{H}, \mathrm{H}-m-\mathrm{Ph}), 8.05$ (d, $J=8.1 \mathrm{~Hz}, 2 \mathrm{H}, \mathrm{H}-m-\mathrm{Ph}), 8.07$ (d, $J=8.3 \mathrm{~Hz}, 2 \mathrm{H}, \mathrm{H}-m-\mathrm{Ph})$, $8.08(\mathrm{~d}, J=8.0 \mathrm{~Hz}, 2 \mathrm{H}, \mathrm{H}-m-\mathrm{Ph}), 8.33$ (d, $J=8.5 \mathrm{~Hz}, 2 \mathrm{H}, \mathrm{H}-o-\mathrm{Ph}), 8.36$ (d, $J=8.3 \mathrm{~Hz}, 2 \mathrm{H}, \mathrm{H}-o-\mathrm{Ph}), 8.37$ $(\mathrm{d}, J=8.0 \mathrm{~Hz}, 2 \mathrm{H}, \mathrm{H}-o-\mathrm{Ph}), 8.43$ (d, $J=8.1 \mathrm{~Hz}, 2 \mathrm{H}, \mathrm{H}-o-\mathrm{Ph}), 8.61-8.69$ (m, 6H, H- $\beta) .{ }^{13} \mathrm{C}$ NMR $(75$

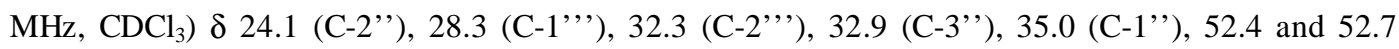
$\left(\mathrm{OCH}_{3}\right), 61.8$ (C-3','), 62.3 (C-4', ), 114.7, 114.8, 119.27, 119.28; 128.18 and 128.23 (C-o-Ph); 128.8, 129.1 (C-o-Ph), 129.8, 130.3 (C-4'); 130.5, 130.8 (C- $\beta$ ), 131.3; 131.59, 131.63, 131.9, 132.6 and 132.7 (C- $\beta) ; 133.00,133.04$ and 133.5 (C-m-Ph); 135.3, 136.0, 140.4, 140.5, 141.3, 141.4, 142.8, 143.9, 145.0, 145.4, 146.3, 154.9 (C-2), 159.5 (C-2 ); 167.1, 167.3 and $168.1(\mathrm{C}=\mathrm{O})$. UV-vis $\left(\mathrm{CHCl}_{3}\right) \lambda_{\max }(\log \varepsilon) 425$ (5.44), 540 (4.21), 579 (4.03). HRMS (ESI) calcd for $\mathrm{C}_{62} \mathrm{H}_{51} \mathrm{~N}_{5} \mathrm{O}_{10} \mathrm{Ni}(\mathrm{M}+\mathrm{H})^{+} 1084.3062$, found 1084.3064.

[3-(2-Hydroxyethyl)-2-(3-hydroxypropyl)-5,10,15,20-tetraphenylpyrido[2,3-

b]porphyrinato]nickel(II), 5a $(21.7 \mathrm{mg}, 68 \%)$. ${ }^{1} \mathrm{H} \mathrm{NMR}\left(300 \mathrm{MHz}, \mathrm{CDCl}_{3}\right) \delta 1.76$ (quintet, $J=6.3 \mathrm{~Hz}$, 2H, H-2'’), 2.89 (t, $J=6.6 \mathrm{~Hz}, 2 \mathrm{H}, \mathrm{H}-1{ }^{\prime \prime}{ }^{\prime}$ ), 2.95 (t, $J=6.3 \mathrm{~Hz}, 2 \mathrm{H}, \mathrm{H}-1$ '’), 3.38 (t, J = 6.3 Hz, 2H, H- 
3'”), 3.78 (t, $J=6.6 \mathrm{~Hz}, 2 \mathrm{H}, \mathrm{H}-2$ ','), 6.96 (s, 1H, H-4'), 7.62-7.80 (m, 12H, H-m,p-Ph), 7.86-7.99 (m, $12 \mathrm{H}, \mathrm{H}-o-\mathrm{Ph}), 8.60-8.72(\mathrm{~m}, 6 \mathrm{H}, \mathrm{H}-\beta) .{ }^{13} \mathrm{C}$ NMR $\left(75 \mathrm{MHz}, \mathrm{CDCl}_{3}\right) \delta 29.7$ (C-2"'), 31.6 (C-1' '), 35.4 (C1'”), 61.9 (C-3"), 62.4 (C-2'"'), 115.4, 115.7, 120.1, 120.2; 126.9, 127.0, 127.1, 127.8, 127.9 and 128.4 (C-m,p-Ph); 128.7 (C-3'); 130.8 (C- $\beta$ ); 131.6, 131.9, and 131.7 (C- $\beta$, C-4'), 132.6 and 132.7 (C- $\beta$ ), 132.9 and $133.5(\mathrm{C}-o-\mathrm{Ph}), 135.0,135.5,140.4,140.7,140.8,141.2,141.8,143.1,144.4,155.1$ (C-2), 158.5 (C2'). UV-vis $\left(\mathrm{CHCl}_{3}\right) \lambda_{\max }(\log \varepsilon) 425$ (5.36), 540 (4.18), 578 (3.95). HRMS (FAB) calcd for $\mathrm{C}_{52} \mathrm{H}_{39} \mathrm{~N}_{5} \mathrm{O}_{2} \mathrm{Ni}(\mathrm{M})^{\circ+}$ calcd 823.2457, found 823.2451.

[3-(2-Hydroxyethyl)-2-(3-hydroxypropyl)-5,10,15,20-tetrakis(3-methoxyphenyl)pyrido[2,3-

b] porphyrinato]nickel(II), $5 \mathbf{b}(17.2 \mathrm{mg}, 48 \%) .{ }^{1} \mathrm{H} \mathrm{NMR}\left(500 \mathrm{MHz}, \mathrm{CDCl}_{3}\right) \delta 1.83$ (quintet, $J=6.4 \mathrm{~Hz}$, 2H, H-2"'), 2.92-2.94 (m, 2H, H-1 '’'), 2.98 (t, $J=6.4$ Hz, 2H, H-1 '), 3.46 (t, $J=6.4 \mathrm{~Hz}, 2 \mathrm{H}, \mathrm{H}-3$ ' '), 3.83 (t, $J=6.5 \mathrm{~Hz}, 2 \mathrm{H}, \mathrm{H}-2$ '"'), 3.88 and $3.89\left(2 \mathrm{~s}, 6 \mathrm{H}, \mathrm{OCH}_{3}\right), 3.91$ (s, 6H, $\left.\mathrm{OCH}_{3}\right), 7.09$ (s, 1H, H-4'), 7.237.24 (m, 3H, H-4-Ph-5,10,15), 7.35 (dd, $J=2.4$ and 8.2 Hz, 1H, H-4-Ph-20), 7.44-7.47 (m, 3H, H-6-Ph20 and $2 \mathrm{H}-2-\mathrm{Ph}-20), 7.53-7.61(\mathrm{~m}, 8 \mathrm{H}, \mathrm{H}-\mathrm{Ph}), 7.65(\mathrm{t}, J=8.0 \mathrm{~Hz}, 1 \mathrm{H}, \mathrm{H}-5-\mathrm{Ph}-20), 8.71$ and $8.72(2 \mathrm{AB}, J$ $=4.5 \mathrm{~Hz}, 4 \mathrm{H}, \mathrm{H}-\beta), 8.75$ and $8.76(\mathrm{AB}, J=5.0 \mathrm{~Hz}, 2 \mathrm{H}, \mathrm{H}-\beta) .{ }^{13} \mathrm{C} \mathrm{NMR}\left(125 \mathrm{MHz}, \mathrm{CDCl}_{3}\right) \delta 29.6(\mathrm{C}-$ 2"'), 31.6 (C-1"'), 35.4 (C-1"'), $55.4\left(\mathrm{OCH}_{3}\right), 55.5\left(\mathrm{OCH}_{3}\right), 62.2$ and $62.3(\mathrm{C}-2$ ", and C-3"'), 113.1 and 113.6 (C-4-Ph-5,10,15), 114.3 (C-4-Ph-20), 115.3, 115.4; 118.6, 118.7 and 119.4 (C-2-Ph-5,10,15); $119.8,120.0 ; 125.9$ (C-6-Ph-20); 125.6, 126.5 and 127.8 (C-5-Ph, C-6-Ph); 128.81 and 128.84 (C-3', C5-Ph-20), 130.9 (C- $\beta$ ); 131.64, 131.67 and 131.70 (C- $\left.\beta, C^{\prime}-4^{\prime}\right) ; 132.6$ and 132.7 (C- $\beta$ ), 135.0, 135.5, 140.7, 141.7, 142.0, 142.5, 143.0, 144.2, 155.0 (C-2), 158.1, 158.5, 158.7 (C-2'), 159.2 (C-3-Ph-20). UVvis $\left(\mathrm{CHCl}_{3}\right) \lambda_{\max }(\log \varepsilon) 425$ (5.41), 540 (4.20), 578 (4.02). HRMS (FAB) calcd for $\mathrm{C}_{56} \mathrm{H}_{48} \mathrm{~N}_{5} \mathrm{O}_{6} \mathrm{Ni}(\mathrm{M}+$ $\mathrm{H})^{+}$944.2958, found 944.2996. Anal. Calcd for $\mathrm{C}_{56} \mathrm{H}_{47} \mathrm{~N}_{5} \mathrm{O}_{6} \mathrm{Ni}$ : C, 71.20; H, 5.01; N, 7.41. Found: $\mathrm{C}$, $70.94 ; \mathrm{H}, 4.75 ; \mathrm{N}, 7.23$.

[3-(2-Hydroxyethyl)-2-(3-hydroxypropyl)-5,10,15,20-tetrakis(4-bromophenyl)pyrido[2,3-

b]porphyrinato]nickel(II), 5c (24.9 mg, 62\%). ${ }^{1} \mathrm{H} \mathrm{NMR}\left(300 \mathrm{MHz}, \mathrm{CDCl}_{3}\right) \delta 1.83$ (quintet, $J=6.5 \mathrm{~Hz}$, 2H, H-2"'), 2.98 (t, $J=6.4 \mathrm{~Hz}, 2 \mathrm{H}, \mathrm{H}-1$ '”'), 3.00 (t, $J=6.5 \mathrm{~Hz}, 2 \mathrm{H}, \mathrm{H}-1$ '), 3.63 (t, $J=6.5 \mathrm{~Hz}, 2 \mathrm{H}, \mathrm{H}-$ 3' '), 3.90 (t, $J=6.4 \mathrm{~Hz}, 2 \mathrm{H}, \mathrm{H}-2$ '”'), 7.10 (s, 1H, H-4'), 7.72-7.86 (m, 14H, H-o-Ph), 7.91 (d, J = 8.2 Hz, $2 \mathrm{H}, \mathrm{H}-\mathrm{o}-\mathrm{Ph}$ ), 8.65-8.69 (m, 6H, H- $\beta$ ). ${ }^{13} \mathrm{C}$ NMR (75 MHz, $\mathrm{CDCl}_{3}$ ) $\delta 29.7$ (C-2'”), 31.8 (C-1'”), 35.3 (C1",'), 62.1 (C-2"'), 62.3 (C-3"'), 114.3, 114.4, 114.5, 119.0, 119.1, 121.7, 122.7, 123.0; 129.5, 130.09, 130.13, 130.2, $130.3(\mathrm{C}-\mathrm{Ph}), 130.8(\mathrm{C}-\beta), 131.2$ (C-4' and C-o-Ph), 131.6 and 132.7 (C- $\beta$ ); 134.2, 134.4, 134.8 and 135.2 (C-Ph); 139.1, 139.46, 139.50, 140.0, 140.7, 140.8, 141.6, 141.7, 143.1, 144.2,154.8 (C2), 159.1 (C-2'). UV-vis $\left(\mathrm{CHCl}_{3}\right) \lambda_{\max }(\log \varepsilon) 425$ (5.44), 540 (4.22), 578 (4.03). HRMS (ESI) calcd for $\mathrm{C}_{52} \mathrm{H}_{36} \mathrm{~N}_{5} \mathrm{O}_{2} \mathrm{Br}_{4} \mathrm{Ni}(\mathrm{M}+\mathrm{H})^{+} 1139.8910$, found 1139.8954 .

[3-(2-Hydroxyethyl)-2-(3-hydroxypropyl)-5,10,15,20-tetrakis(4-carboxymethylphenyl)pyrido[2,3b]porphyrinato]nickel(II), 5d (20.1 mg, 50\%). ${ }^{1} \mathrm{H} \mathrm{NMR}\left(300 \mathrm{MHz}, \mathrm{CDCl}_{3}\right) \delta 1.69$ (quintet, $J=6.6 \mathrm{~Hz}$, 2H, H-2"'), 2.87-2.92 (m, 4H, H-1"' and H-1 ', '), 3.49 (t, $J=6.6 \mathrm{~Hz}, 2 \mathrm{H}, \mathrm{H}-3$ "), 3.77 (t, $J=6.5 \mathrm{~Hz}, 2 \mathrm{H}$, H-2'”'), 4.08, 4.09 and 4.20 (3s, $12 \mathrm{H}, 4 \mathrm{CO}_{2} \mathrm{CH}_{3}$ ), 7.06 (s, $1 \mathrm{H}, \mathrm{H}-4$ '), 7.97 (d, $J=8.3 \mathrm{~Hz}, 2 \mathrm{H}, \mathrm{H}-m-\mathrm{Ph}$ ), 8.03-8.08 (m, 6H, H-m-Ph), 8.33-8.38 (m, 6H, H-o-Ph), 8.43 (d, $J=8.3$ Hz, 2H, H-o-Ph), 8.61-8.65 (m, $4 \mathrm{H}, \mathrm{H}-\beta), 8.62$ and $8.65(\mathrm{AB}, J=5.0 \mathrm{~Hz}, 2 \mathrm{H}, \mathrm{H}-\beta) .{ }^{13} \mathrm{C} \mathrm{NMR}\left(75 \mathrm{MHz}, \mathrm{CDCl}_{3}\right) \delta 29.9$ (C-2'”), 31.5 (C1'”), 35.4 (C-1'”), 52.4 and $52.6\left(\mathrm{OCH}_{3}\right), 62.2$ (C-2'”'), 62.5 (C-3"'), 114.5, 114.9, 119.3, 119.4; 128.2,

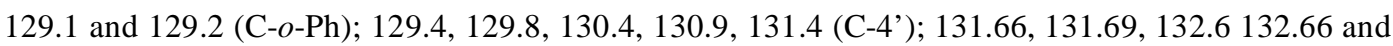


132.7 (C- $\beta$ ), 133.0 and 133.5 (C-m-Ph), 135.1, 135.7, 140.4, 140.5, 141.38, 141.42, 142.9, 143.9, 144.9, 145.2, 146.1, 155.2 (C-2), 159.2 (C-2'); 167.1 and 167.8 (C=O). UV-vis $\left(\mathrm{CHCl}_{3}\right) \lambda_{\max }(\log \varepsilon) 425$ (5.40), 540 (4.20), 579 (4.02). HRMS (ESI) calcd for $\mathrm{C}_{60} \mathrm{H}_{36} \mathrm{~N}_{5} \mathrm{O}_{10} \mathrm{Ni}(\mathrm{M}+\mathrm{H})^{+}$1056.2749, found 1056.2776.

\section{Experimental Procedures and Spectroscopic data for 6}

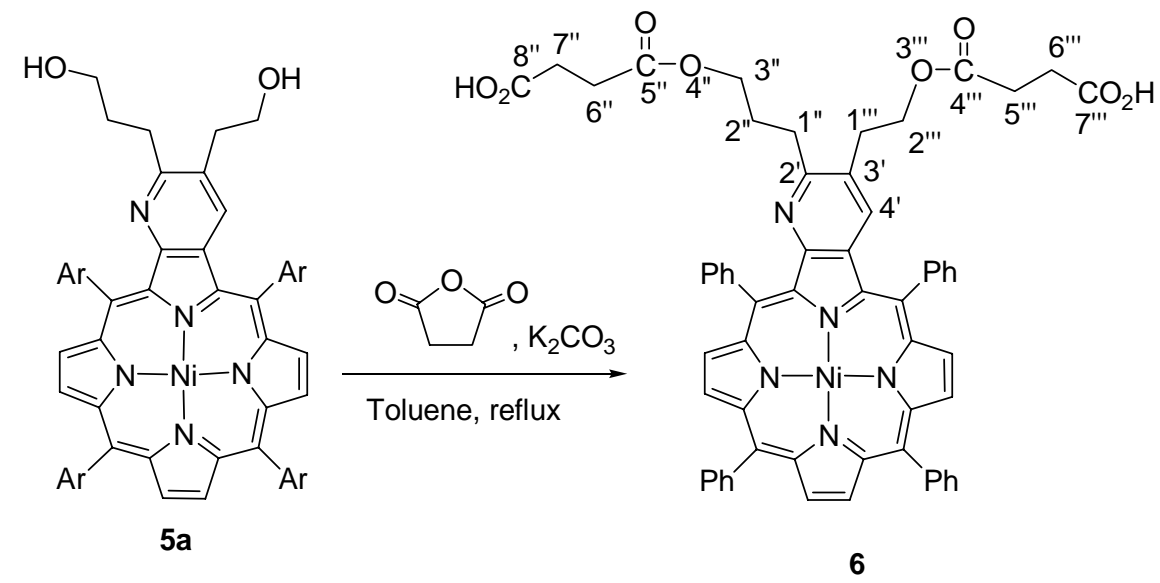

Succinic anhydride $(18.3 \mathrm{mg}, 0.180 \mathrm{mmol})$ and potassium carbonate $(20.5 \mathrm{mg}, 0.209 \mathrm{mmol})$ were added to a stirred solution of $\mathbf{5 a}(15.0 \mathrm{mg}, 0.018 \mathrm{mmol})$ in dry toluene $(2 \mathrm{~mL})$. The mixture was heated at reflux, under a nitrogen atmosphere, until the complete conversion of the starting material ( 20 hours, observed by TLC). After reaching room temperature the reaction mixture was neutralized with aqueous saturated citric acid solution, washed with distillated water, extracted with chloroform and dried over $\mathrm{Na}_{2} \mathrm{SO}_{4}$. After solvent removal under reduced pressure and precipitation from dichloromethane: methanol compound 6 was obtained pure as a purple solid in $92 \%$ yield $(16.9 \mathrm{mg}) .{ }^{1} \mathrm{H} \mathrm{NMR}\left(300 \mathrm{MHz}, \mathrm{CDCl}_{3}\right) \delta 1.73-1.82(\mathrm{~m}$, 2H, H-2'”), 2.61-2.73 (m, 8H, H-6', H-7', H-5', ', H-6' ' '), 2.81 (t, J= 7.4 Hz, 2H, H-1 ' '), 2.89 (t, J = 8.0 Hz, 2H, H-1', '), 4.13 (t, J = 5.4 Hz, 2H, H-3' '), 4.21 (t, J=8.0 Hz, 2H, H-2', '), 6.86 (s, 1H, H-4'), 7.628.01 (m, 20H, H-o, $m, p-\mathrm{Ph}), 8.66$ (AB, $J=5.2 \mathrm{~Hz}, 2 \mathrm{H}, \mathrm{H}-\beta), 8.67$ (s, $2 \mathrm{H}, \mathrm{H}-\beta), 8.71$ and 8.74 (AB, $J=5.0$ $\mathrm{Hz}, 2 \mathrm{H}, \mathrm{H}-\beta) .{ }^{13} \mathrm{C} \mathrm{NMR}\left(75 \mathrm{MHz}, \mathrm{CDCl}_{3}\right.$ ) $\delta 24.6$ (C-2'”); 29.37, 29.40 and 29.7 (C-6', C-7', C-5', , C6', ); 30.2 (C-1','), 30.8 (C-1'’), 62.8 (C-2','), 65.3 (C-3', ), 115.5, 115.7, 117.5, 120.0, 120.1, 120.6; 126.8 and $127.0(\mathrm{C}-m-\mathrm{Ph}) ; 127.6 ; 127.8 ; 127.9,128.0,128.2$ and $128.7(\mathrm{C}-p-\mathrm{Ph}) ; 129.3\left(\mathrm{C}^{\prime}{ }^{\prime}\right), 130.8(\mathrm{C}-$ $\beta), 131.5,131.6 ; 131.7,132.5$ and 132.6 (C- $\beta) ; 132.8$ and 133.5 (C-o-Ph); 135.1, 135.7, 138.4, 139.3, 140.4, 140.56, 140.62, 140.8, 140.9, 141.4, 141.7, 142.0, 142.8, 143.0, 144.4, 155.0 (C-2), 157.2 (C-2'), 171.4 (C-4','), 172.1 (C-5'”), 179.5 (C-8', and C-7','). UV-vis $\left(\mathrm{CHCl}_{3}\right) \lambda_{\max }(\log \varepsilon) 425$ (5.16), 540 (4.14), 579 (3.90). HRMS (ESI) calcd for $\mathrm{C}_{60} \mathrm{H}_{48} \mathrm{~N}_{5} \mathrm{O}_{8} \mathrm{Ni}(\mathrm{M}+\mathrm{H})^{+}$1024.2851, found 1024.2833. 


\section{Experimental Procedures and Spectroscopic data for 7}

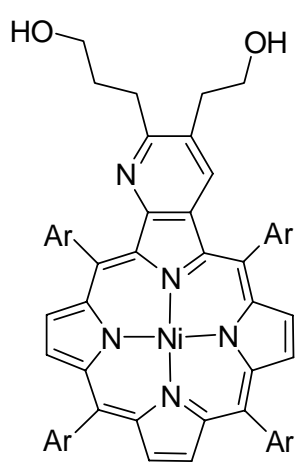

$5 a$

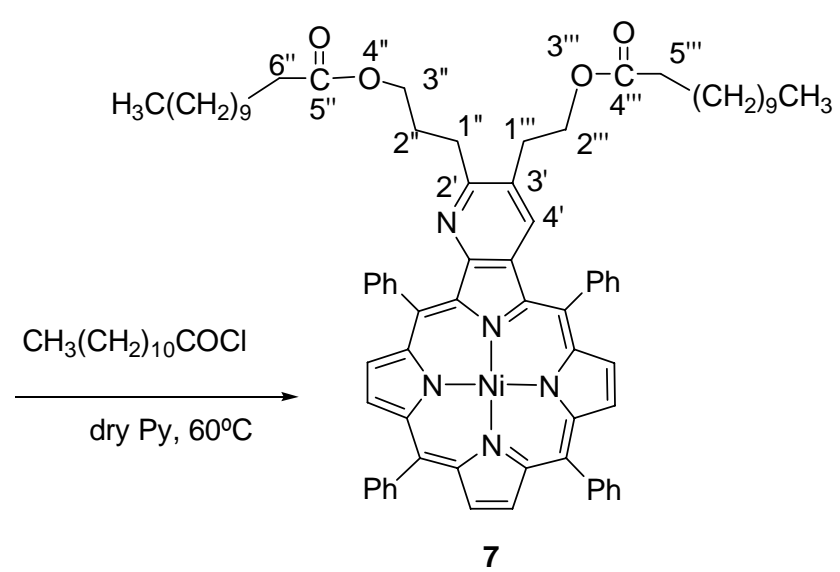

To a stirred solution of $\mathbf{5 a}(16.1 \mathrm{mg}, 0.0195 \mathrm{mmol})$ in dry pyridine $(2 \mathrm{~mL})$ it was added dodecanoyl chloride $(50 \mu \mathrm{L}, 0.104 \mathrm{mmol})$. The reaction mixture was protected against moisture and stirred at $60^{\circ} \mathrm{C}$ until the complete consumption of the starting porphyrin (20 hours, observed by TLC). After reaching room temperature the reaction mixture was neutralized with aqueous saturated citric acid solution, washed with distillated water, extracted with chloroform and dried over $\mathrm{Na}_{2} \mathrm{SO}_{4}$. After solvent removal under reduced pressure the obtained residue was dissolved in dichloromethane and purified by column chromatography using dichloromethane as the eluent. Compound 7 was obtained pure as purple crystals from dichloromethane: methanol (22.2 mg, 96\%). ${ }^{1} \mathrm{H} \mathrm{NMR}\left(300 \mathrm{MHz}, \mathrm{CDCl}_{3}\right) \delta 0.82(\mathrm{t}, J=6.9 \mathrm{~Hz}, 3 \mathrm{H}$, $\left.\mathrm{CH}_{3}\right), 0.85\left(\mathrm{t}, J=6.7 \mathrm{~Hz}, 3 \mathrm{H}, \mathrm{CH}_{3}\right), 1.13-1.25$ (m, 36H, $\mathrm{CH}_{2}$ ), 1.52-1.68 (m, 4H, H-7' ', H-6' ' '), 1.76-1.85 (quintet, $J=7.0 \mathrm{~Hz}, 2 \mathrm{H}, \mathrm{H}-2$ ' '), 2.26 (t, $J=7.6 \mathrm{~Hz}, 2 \mathrm{H}, \mathrm{H}-5$ ', '), 2.32 (t, $J=7.6 \mathrm{~Hz}, 2 \mathrm{H}, \mathrm{H}-6$ ' '), 2.86 (t, $J$ $=7.0 \mathrm{~Hz}, 2 \mathrm{H}, \mathrm{H}-1$ ' '), 2.94 (t, $J=7.1 \mathrm{~Hz}, 2 \mathrm{H}, \mathrm{H}-1$ ', '), 4.05 (t, $J=7.0 \mathrm{~Hz}, 2 \mathrm{H}, \mathrm{H}-3$ '’), 4.17 (t, $J=7.1 \mathrm{~Hz}$, 2H, H-2',' ), 7.62-8.01 (m, 20H, H- $-m, p-\mathrm{Ph}), 8.67$ (AB, $J=4.5 \mathrm{~Hz}, 2 \mathrm{H}, \mathrm{H}-\beta), 8.68$ and 8.70 (AB, $J=5.0$ $\mathrm{Hz}, 2 \mathrm{H}, \mathrm{H}-\beta), 8.71$ and $8.72(\mathrm{AB}, J=5.1 \mathrm{~Hz}, 2 \mathrm{H}, \mathrm{H}-\beta) .{ }^{13} \mathrm{C} \mathrm{NMR}\left(75 \mathrm{MHz}, \mathrm{CDCl}_{3}\right) \delta 14.07$ and 14.10 $\left(\mathrm{CH}_{3}\right), 22.6$ and $22.7\left(\mathrm{CH}_{2}\right), 24.9$ and 25.1 (C-7', , C-6',' '), 26.0 (C-2'’ '); 29.1, 29.2, 29.3, 29.4, 29.5, 29.6

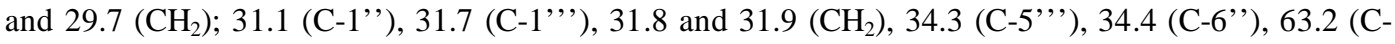
2','), 64.4 (C-3'’), 115.5, 115.7, 120.0, 120.1; 126.87 and 126.94 (C-m-Ph); 127.79, 127.81 and 127.9 (Cp-Ph); 128.6, 130.8 (C- $\beta$ ), 131.3 (C-4'); 131.56, 131.59, 131.7, 132.5 and 132.6 (C- $\beta$ ); 132.7, 132.8 and $133.5(\mathrm{C}-o-\mathrm{Ph}) ; 135.1,135.7,140.4,140.6,140.7,140.8,141.3,141.69,141.71,143.1,144.4,155.4$ (C2), 157.5 (C-2'), 173.6 (C-4','), 174.0 (C-5'’). UV-vis $\left(\mathrm{CHCl}_{3}\right) \lambda_{\max }(\log \varepsilon) 425$ (5.44), 540 (4.22), 578 (4.03). Maldi MS for $\mathrm{C}_{76} \mathrm{H}_{64} \mathrm{~N}_{5} \mathrm{NiO}_{4}\left([\mathrm{M}+\mathrm{H}]^{+}\right) 1188$

\section{Single-crystal X-ray Diffraction Studies of 7}

Suitable single-crystals of compound 7 were manually harvested from the crystallisation vial and mounted on a Hampton Research CryoLoop using FOMBLIN Y perfluoropolyether vacuum oil (LVAC 25/6) purchased from Aldrich, ${ }^{3}$ with the help of a Stemi 2000 stereomicroscope equipped with Carl Zeiss lenses. Data were collected at 180(2)K on a Bruker X8 Kappa APEX II charge-coupled device (CCD) area-detector diffractometer $\left(\mathrm{Cu} \mathrm{K} \mathrm{K}_{\alpha}\right.$ graphite-monochromated radiation, $\lambda=1.54178 \AA$ ) controlled by the APEX2 software package, ${ }^{4}$ and equipped with an Oxford Cryosystems Series 700 cryostream monitored 
remotely using the software interface Cryopad. ${ }^{5}$ Images were processed using the software package SAINT $+{ }^{6}$ and data were corrected for absorption by the multi-scan semi-empirical method implemented in SADABS. ${ }^{7}$

It is of considerable importance to note that the available crystalline material for the crystallographic studies was uniformly composed of very thin plates. The largest plate which could be selected for singlecrystal data collection $(0.18 \times 0.16 \times 0.04 \mathrm{~mm})$ still diffracted very weakly at high angles with a very low value of $\sin (\theta) / \lambda$ for the merged data set. Indeed, reflections could only be detected up to $0.95 \AA$ of resolution (even for longer frame exposures), hence the low data completeness (90.7\%) of the data set used for structure solution and refinement.

Analysis of the collected data with the XPREP interface strongly pointed to a centrosymmetric structure based on the calculated mean $\left|\mathrm{E}^{2}-1\right|$ value, with the systematic absences further indicating space group $P \overline{\mathbf{1}}$ as the best candidate. However, this space group did not produce a reliable initial structural model, either by using direct methods or Patterson synthesis. Therefore, the structure was instead solved in space group $P 1$ using the direct methods implemented with SHELXS- $97,{ }^{8}$ which ultimately allowed the immediate location of majority of atoms for two crystallographically independent porphyrin molecules. All the remaining non-hydrogen atoms were directly located from difference Fourier maps calculated from successive full-matrix least squares refinement cycles on $F^{2}$ using SHELXL-97. ${ }^{9}$ At this stage, a visual inspection of the two crystallographically independent porphyrin molecules strongly suggested that, as initially expected, they could be related by inversion. In fact, ADDSYM ${ }^{10,11}$ routines provided with PLATON $^{12,13}$ unambiguously identified $P \overline{1}$ as the correct space group for porphyrin 7 . Structural refinement proceeded in $P \overline{1}$ with all non-hydrogen atoms being successfully refined using anisotropic displacement parameters (see Figure S18).

The two $\mathrm{CO}\left(\mathrm{CH}_{2}\right)_{10} \mathrm{CH}_{3}$ chains bound to the hydroxyalkyl groups of the pyridine-fused moiety were found to be severely affected by thermal disorder, in particular the $\mathrm{C}(41) \rightarrow \mathrm{C}(52)$ chain as depicted in Figure S18. In order to ensure chemically reasonable geometries for the carbon atoms forming these chains, the $\mathrm{C}-\mathrm{C}$ direct bonds were restrained to a common refineable value for both chains which ultimately refined to $1.500(2) \AA$.

Hydrogen atoms attached to carbon were located at their idealised positions using appropriate HFIX instructions in SHELXL: 43 for the aromatic carbon atoms associated with the phenyl groups, for the six $\beta$-pyrrolic carbon atoms and also the carbon atom of the pyridine-fused moiety; 137 for the terminal methyl groups of alkyl chains. These hydrogen atoms were included in subsequent refinement cycles in riding-motion approximation with isotropic thermal displacements parameters $\left(U_{\text {iso }}\right)$ fixed at 1.2 or 1.5 (only for the $-\mathrm{CH}_{3}$ moieties) times $U_{e q}$ of the carbon atom to which they are attached.

The last difference Fourier map synthesis showed the highest peak $\left(0.668 \mathrm{e}^{-3}\right)$ and deepest hole $\left(-0.555 \mathrm{e}^{-3}\right)$ located at $1.21 \AA$ from $\mathrm{O}(1)$ and $0.69 \AA$ from $\mathrm{C}(44)$, respectively.

Information concerning crystallographic data collection and structure refinement details is summarised in Table S1. Selected bond lengths and angles for the nickel coordination environment are supplied in Table S2. Structural representations were performed with the visualization software package Crystal Diamond. ${ }^{14}$ 
Crystallographic data (excluding structure factors) for the structure reported in this paper have been deposited with the Cambridge Crystallographic Data Centre as supplementary publication no. CCDC-633482. Copies of the data can be obtained free of charge on application to CCDC, 12 Union Road, Cambridge CB2 2EZ, U.K. FAX: (+44) 1223 336033. E-mail: deposit@ccdc.cam.ac.uk. 


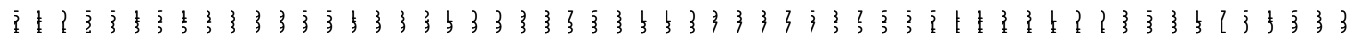

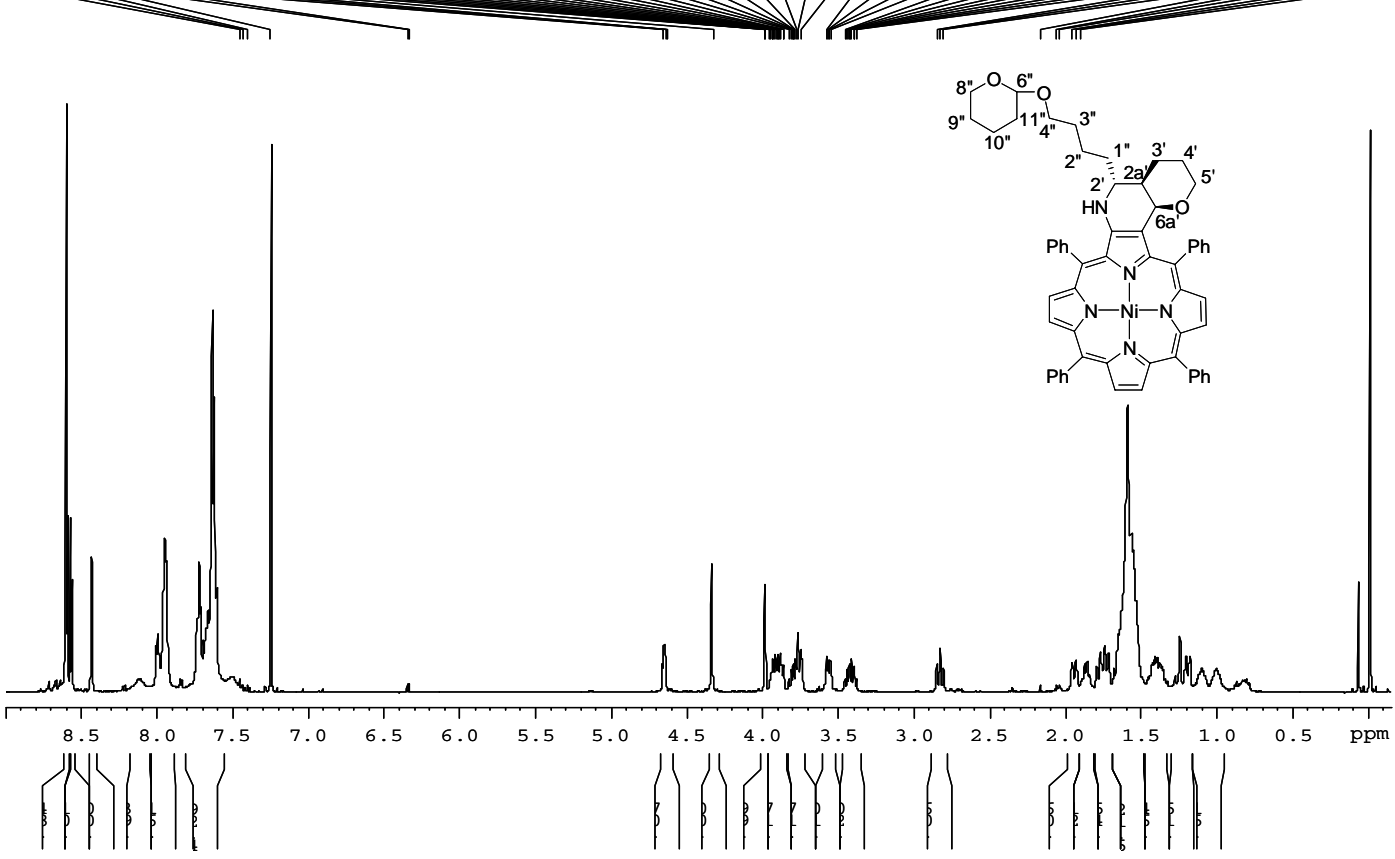

Figure S1. ${ }^{1} \mathrm{H}$ NMR of compound $\mathbf{2 a}$.

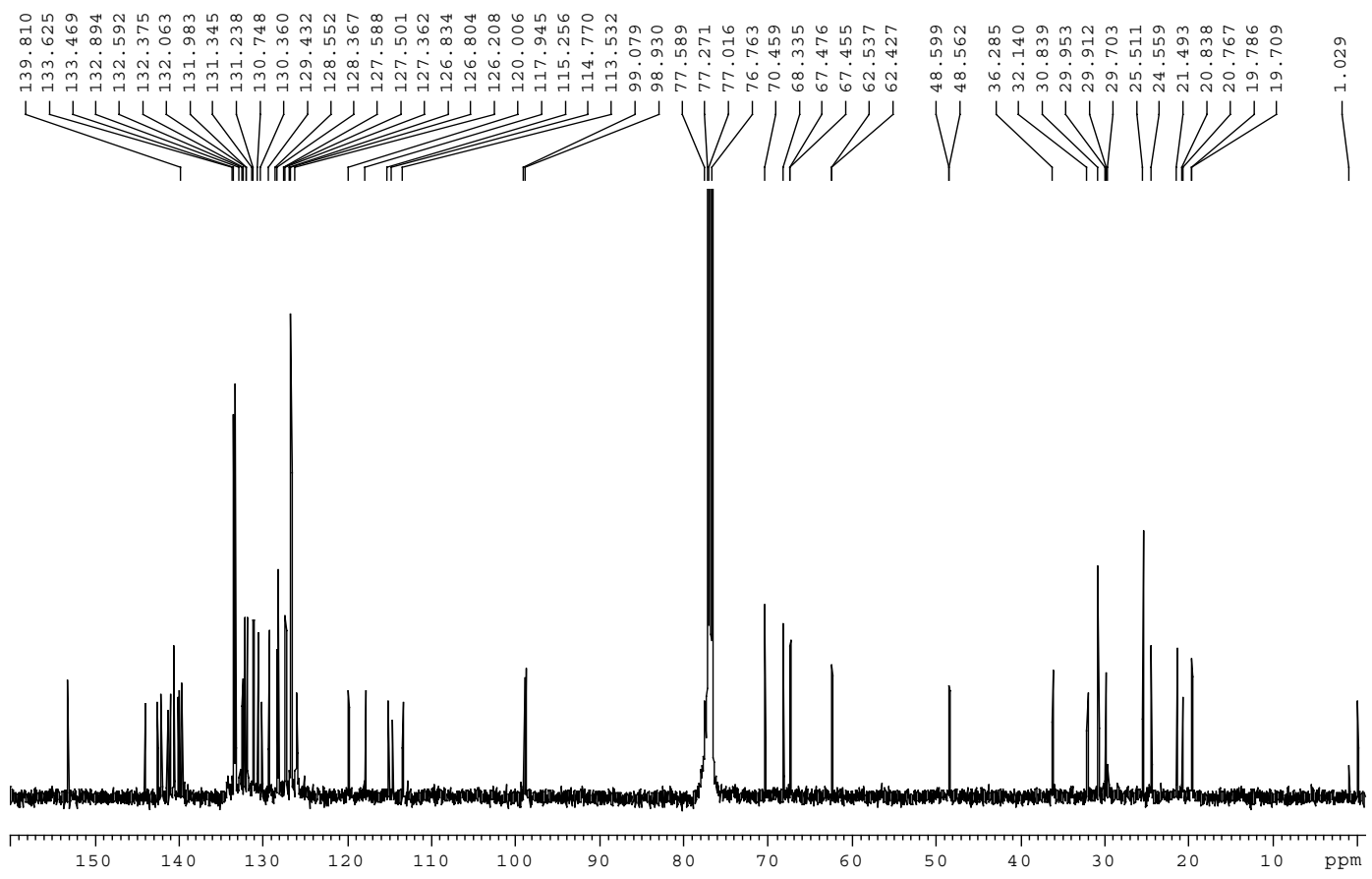

Figure S2. ${ }^{13} \mathrm{C}$ NMR of compound $\mathbf{2 a}$. 


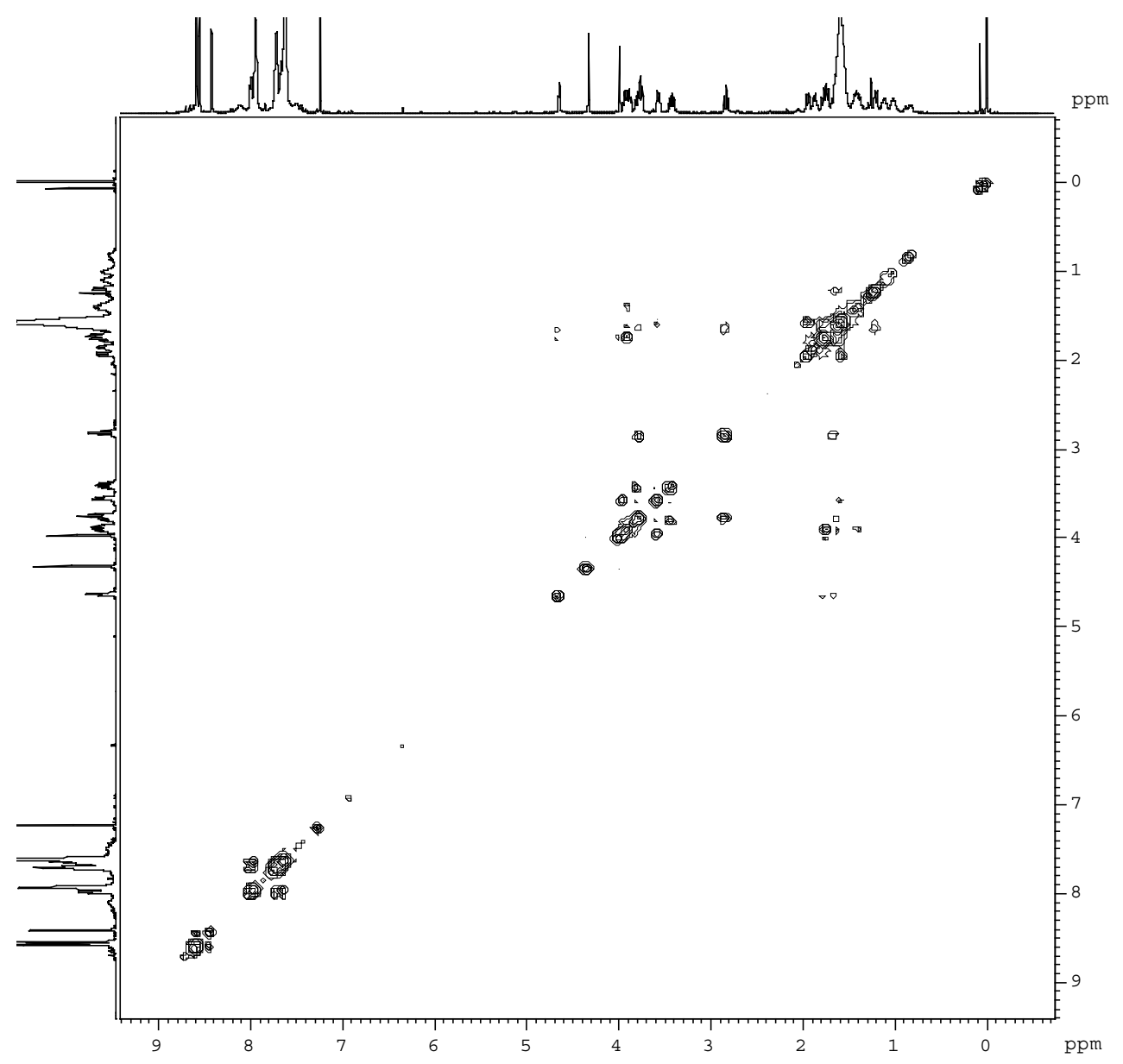

Figure S3. COSY of compound 2a. 


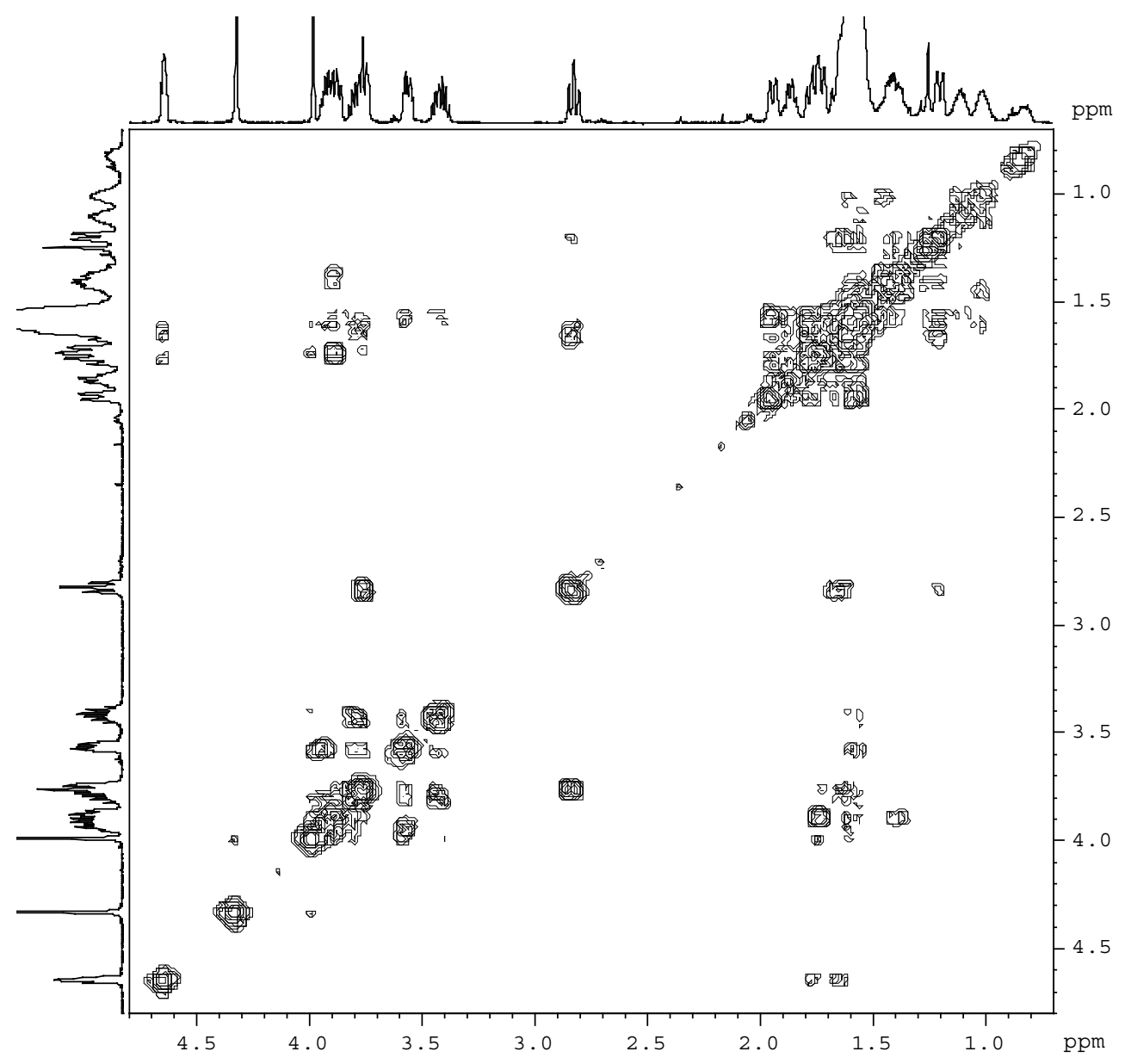

Figure S4. Partial COSY of compound 2a. 


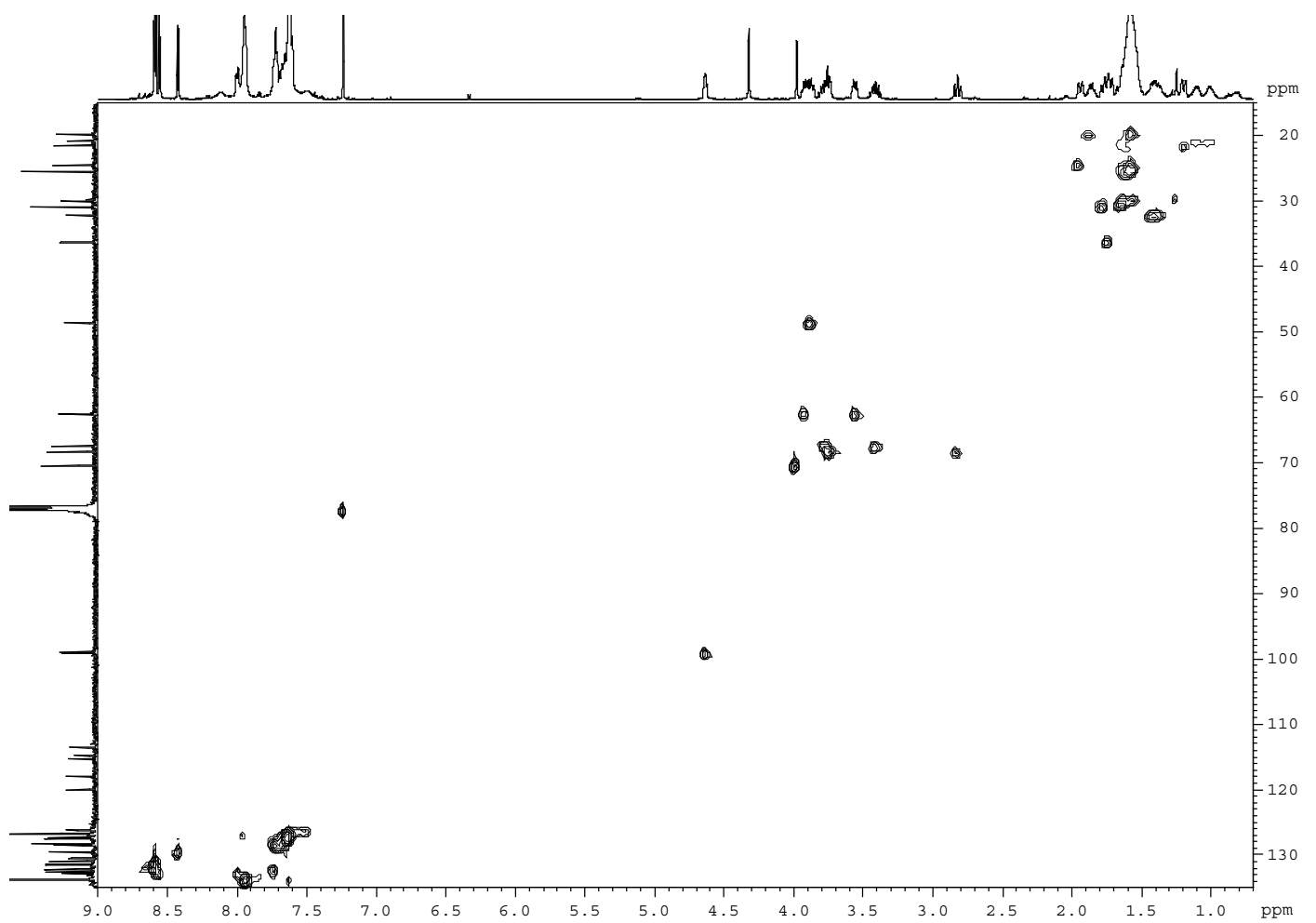

Figure S5. HSQC of compound 2a.

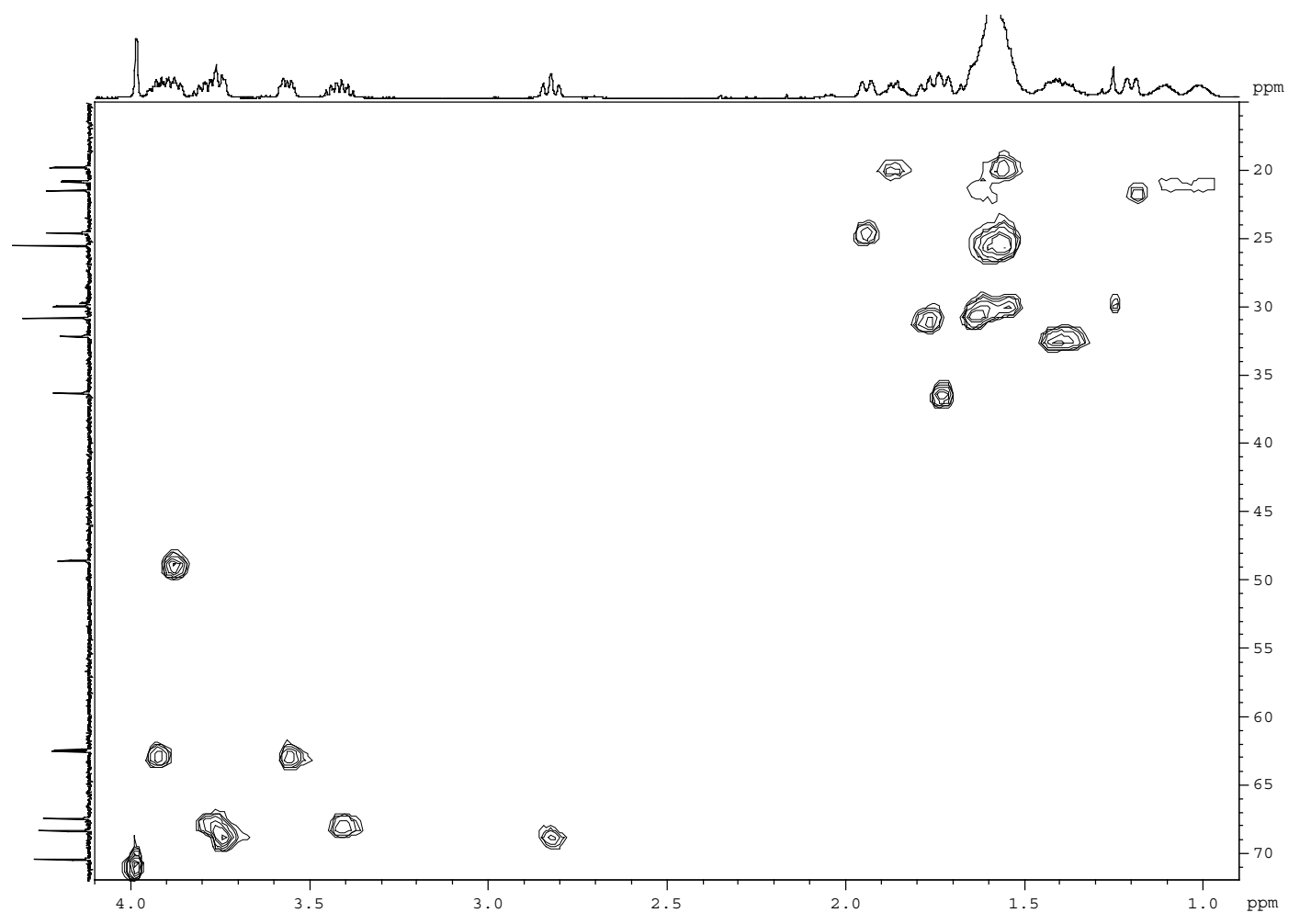

Figure S6. Partial HSQC of compound 2a. 


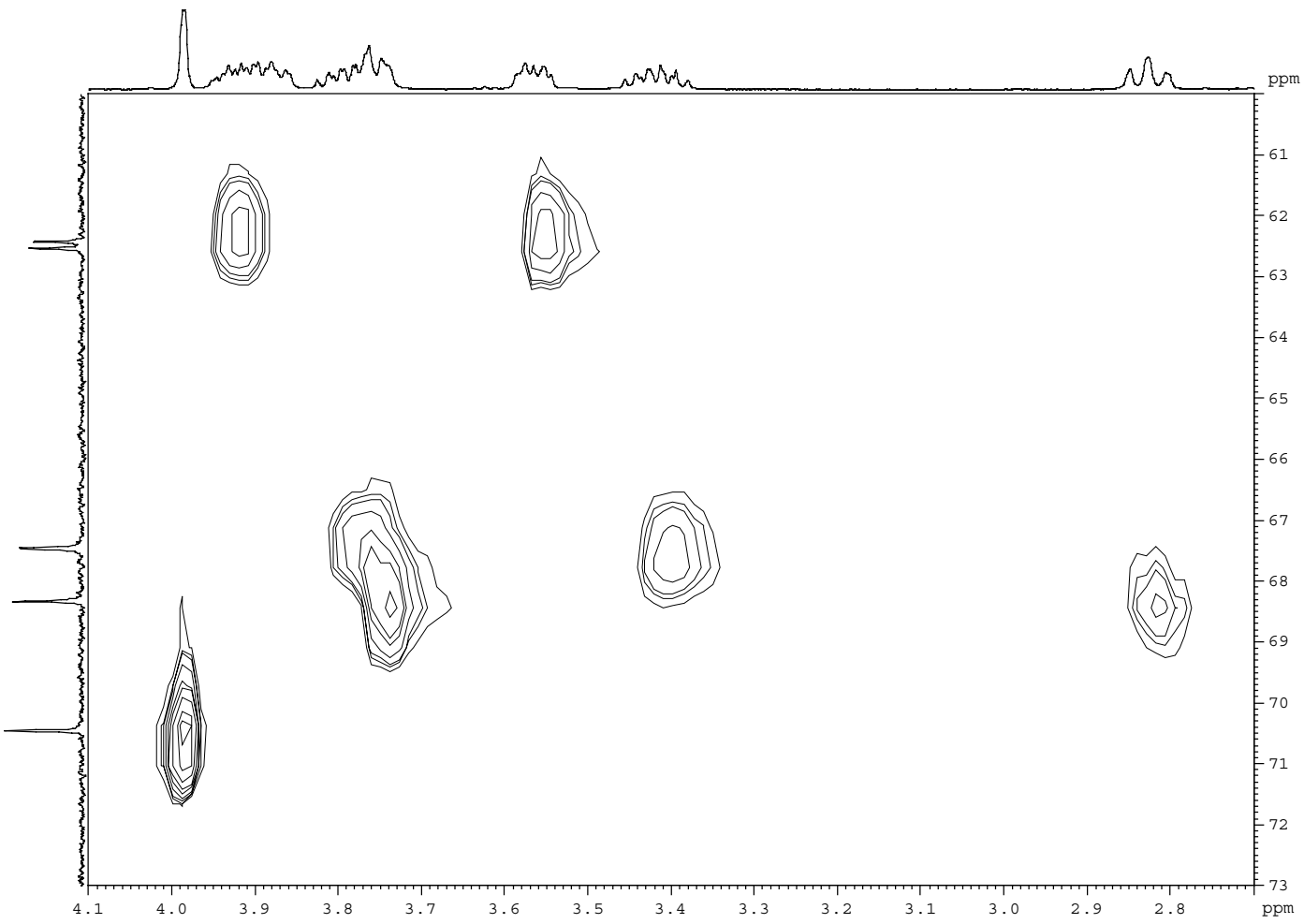

Figure S7. Partial HSQC of compound 2a

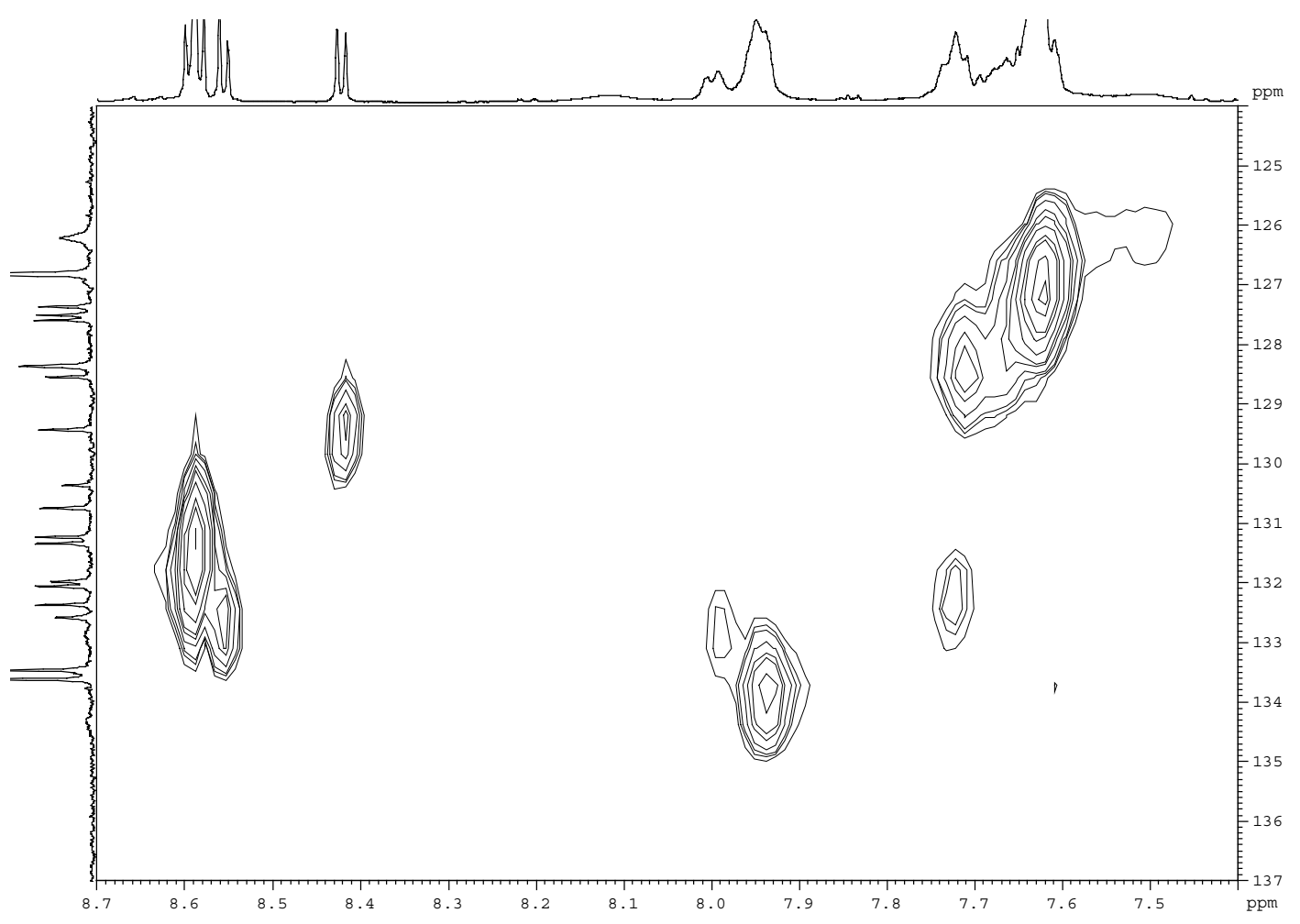

Figure S8. Partial HSQC of compound 2a 


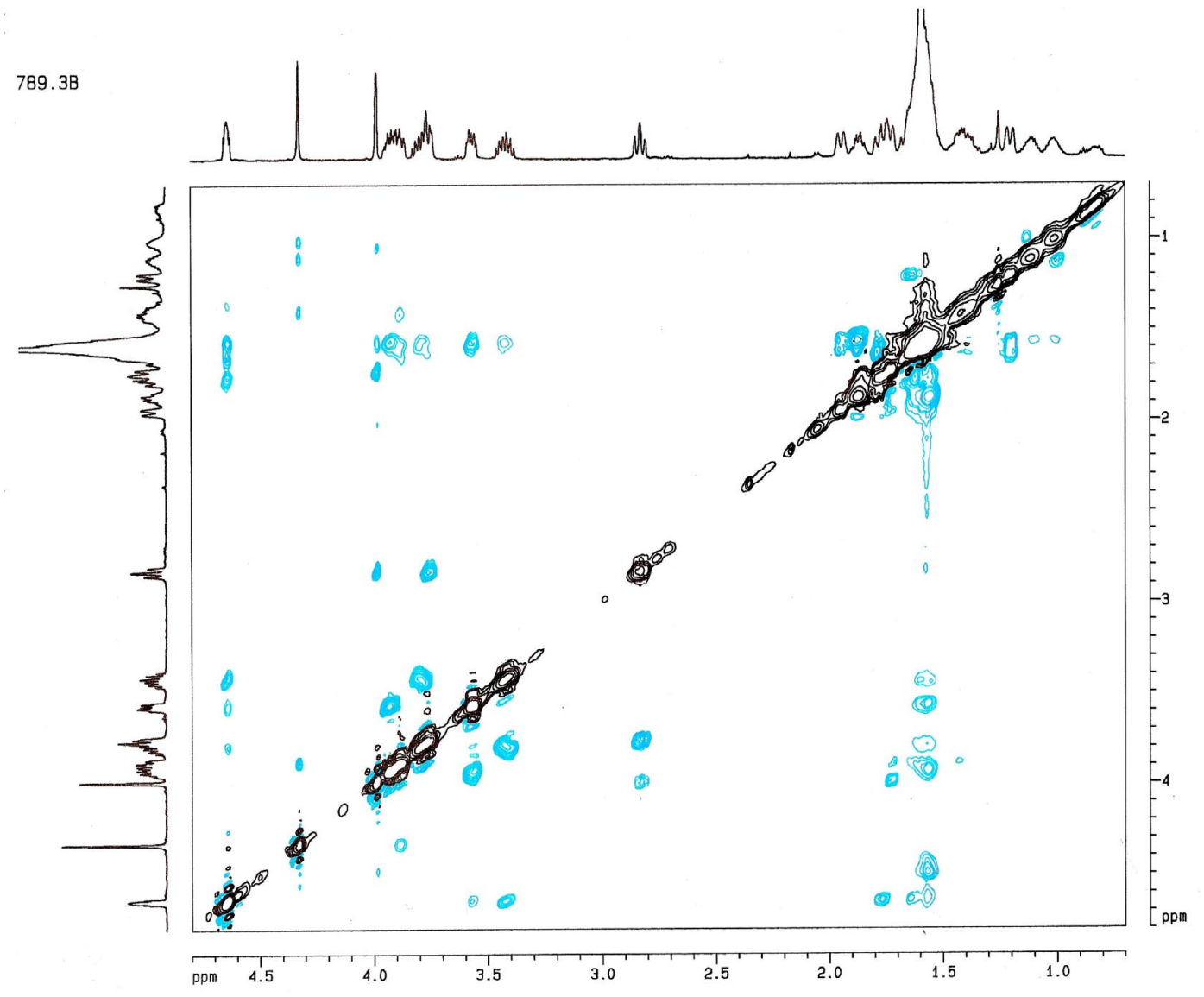

Figure S9. Partial NOESY of compound 2a 


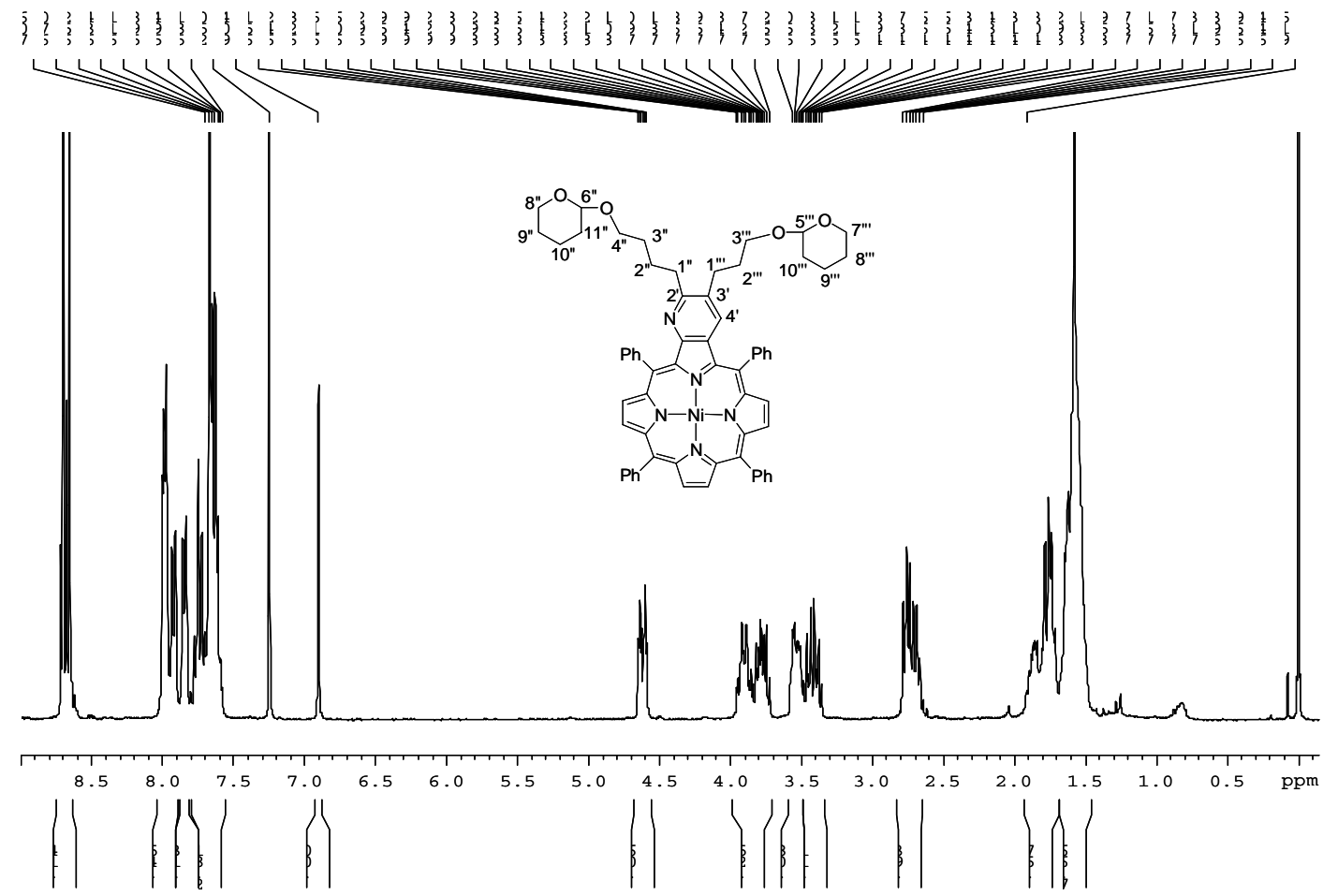

Figure S10. ${ }^{1} \mathrm{H}$ NMR of compound 3a

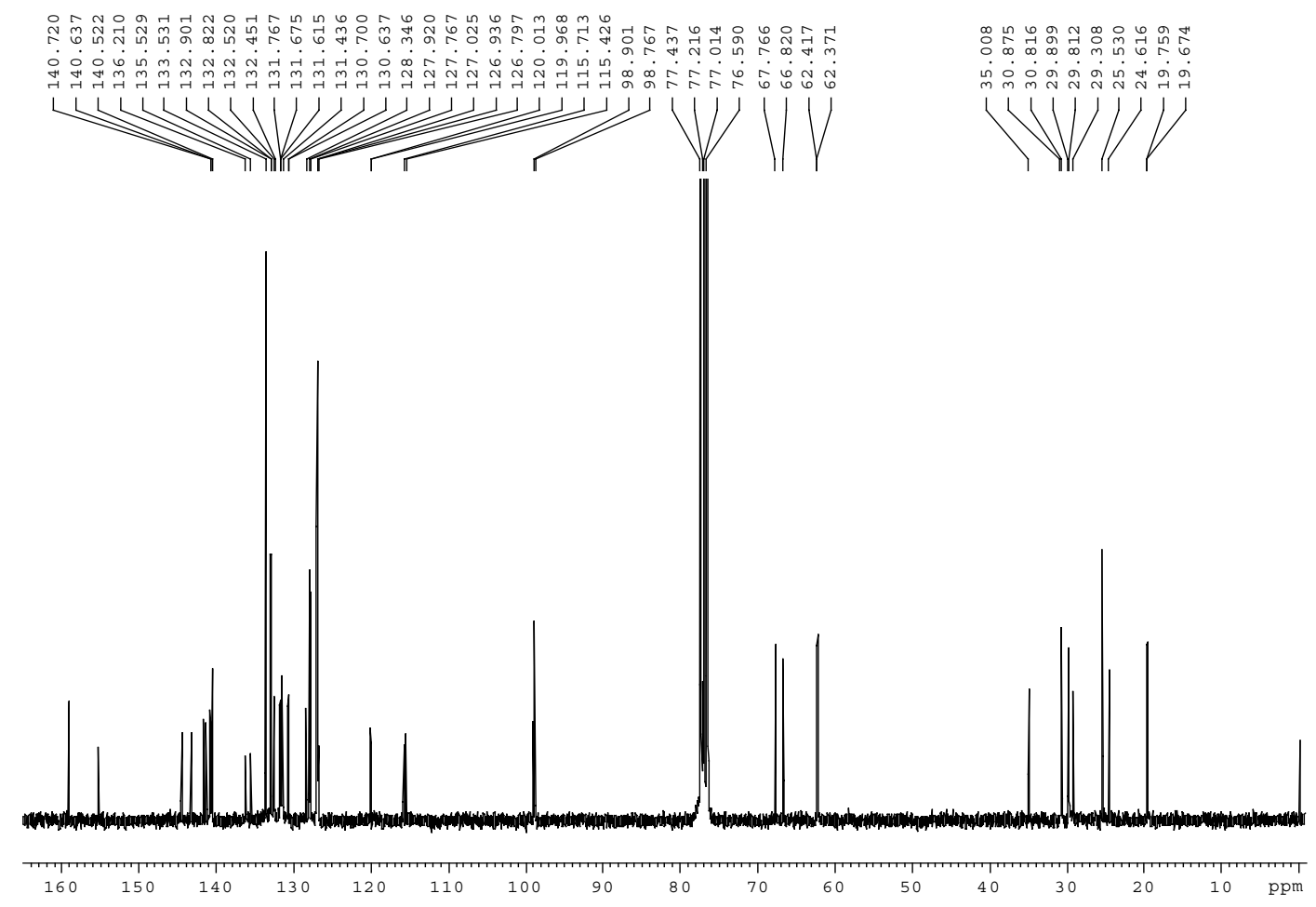

Figure S11. ${ }^{13} \mathrm{C}$ NMR of compound $\mathbf{3 a}$ 


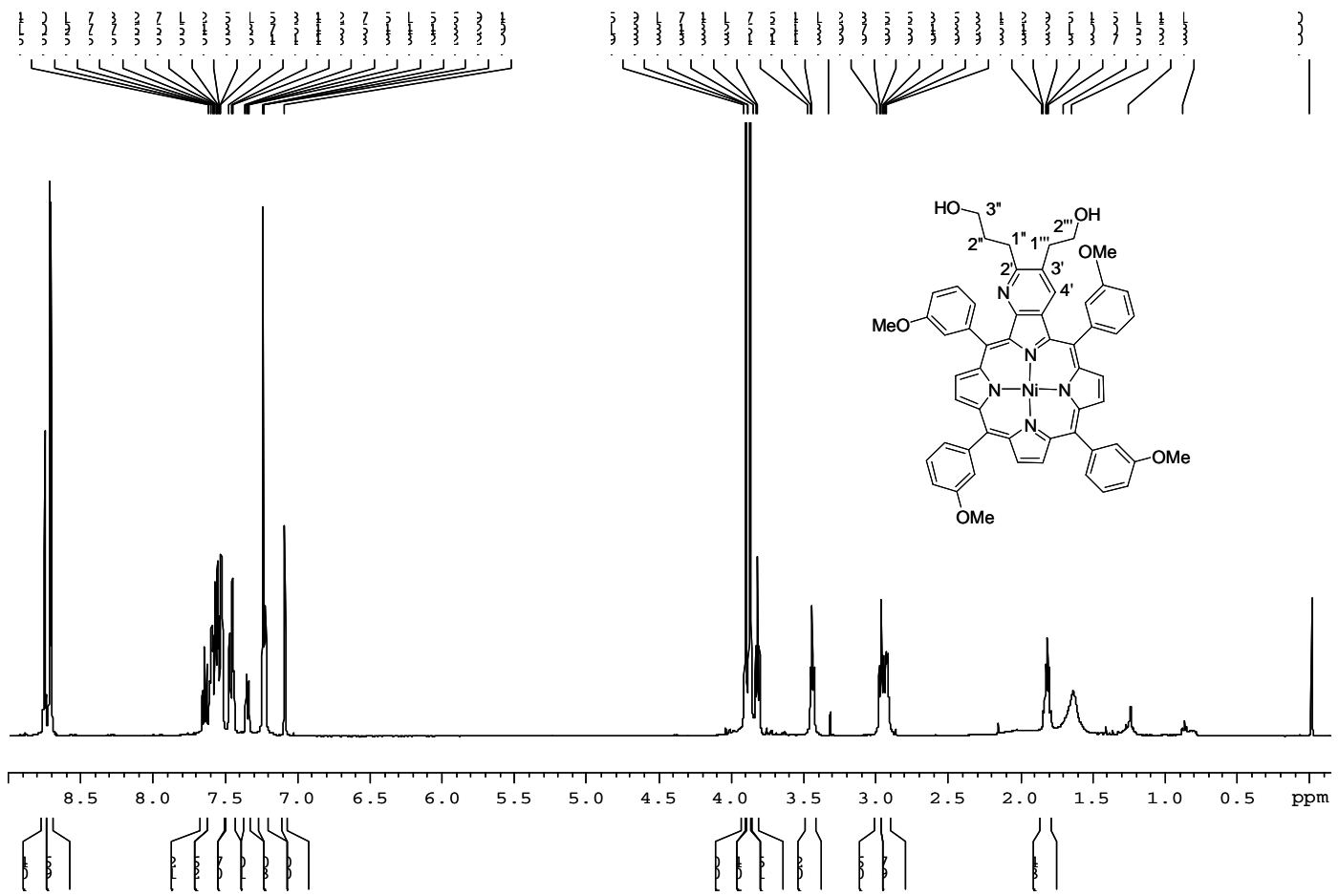

Figure S12. ${ }^{1} \mathrm{H}$ NMR of compound $\mathbf{5 b}$.

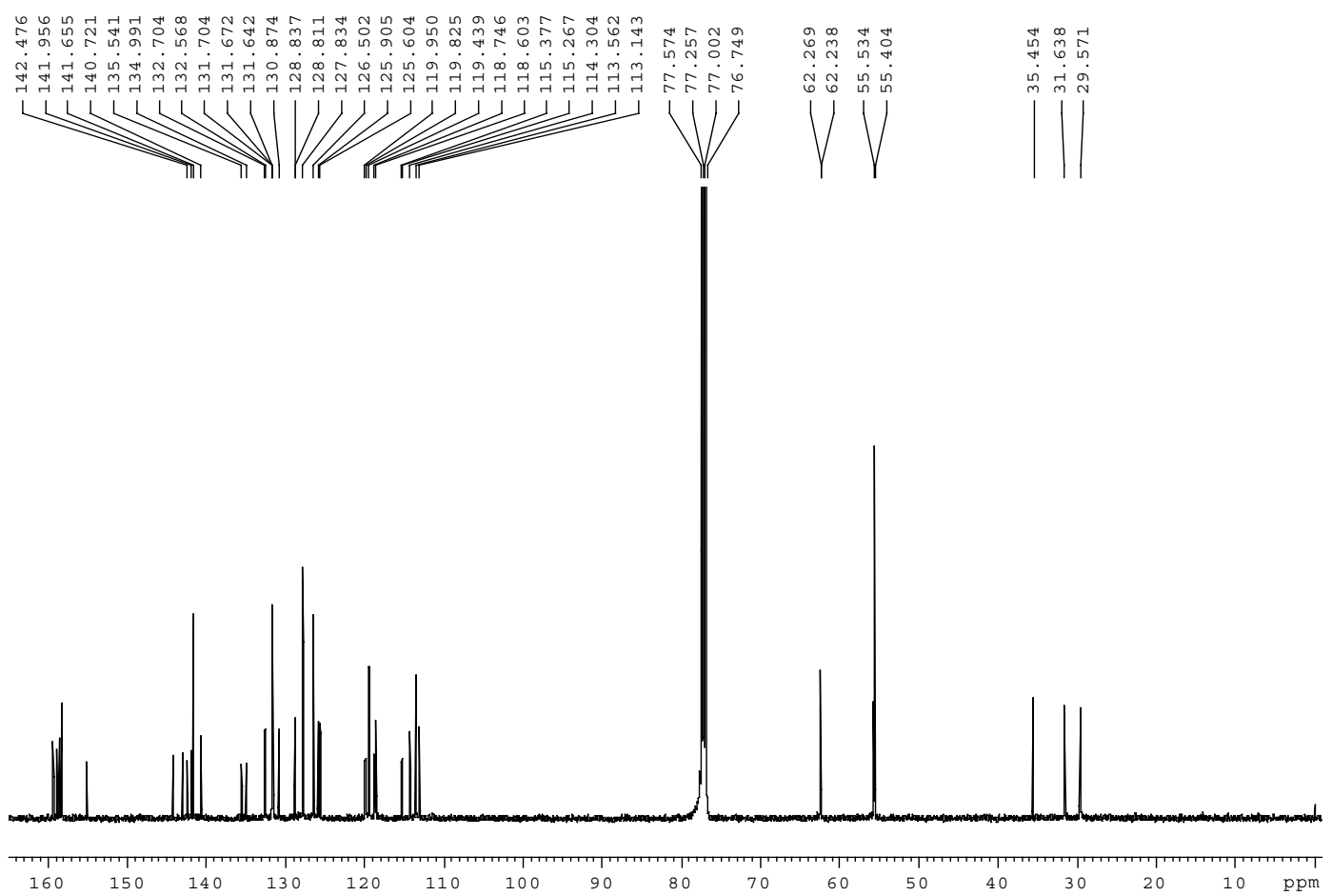

Figure S13. ${ }^{13} \mathrm{C}$ NMR of compound $\mathbf{5 b}$. 


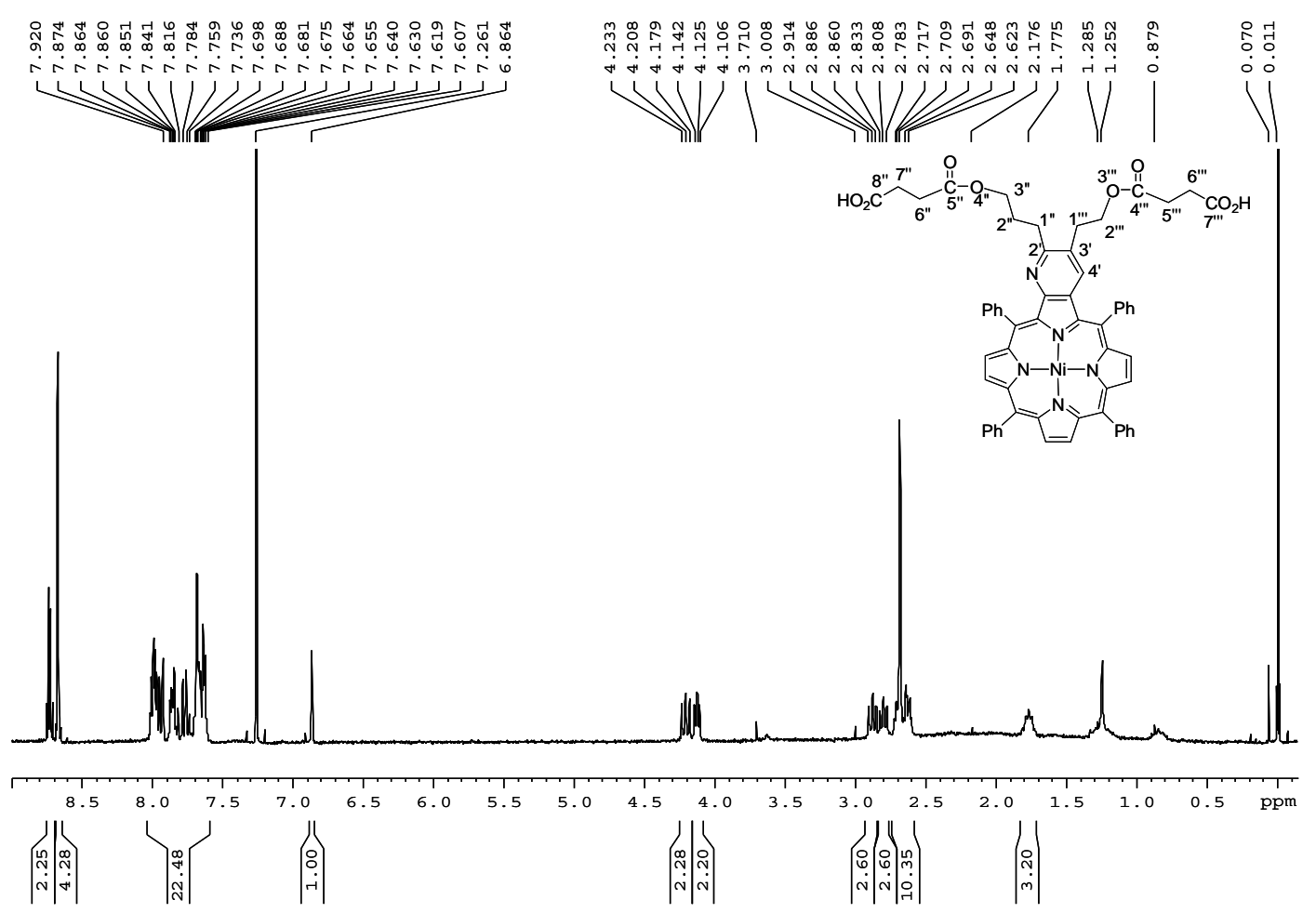

Figure S14. ${ }^{1} \mathrm{H}$ NMR of compound 6.

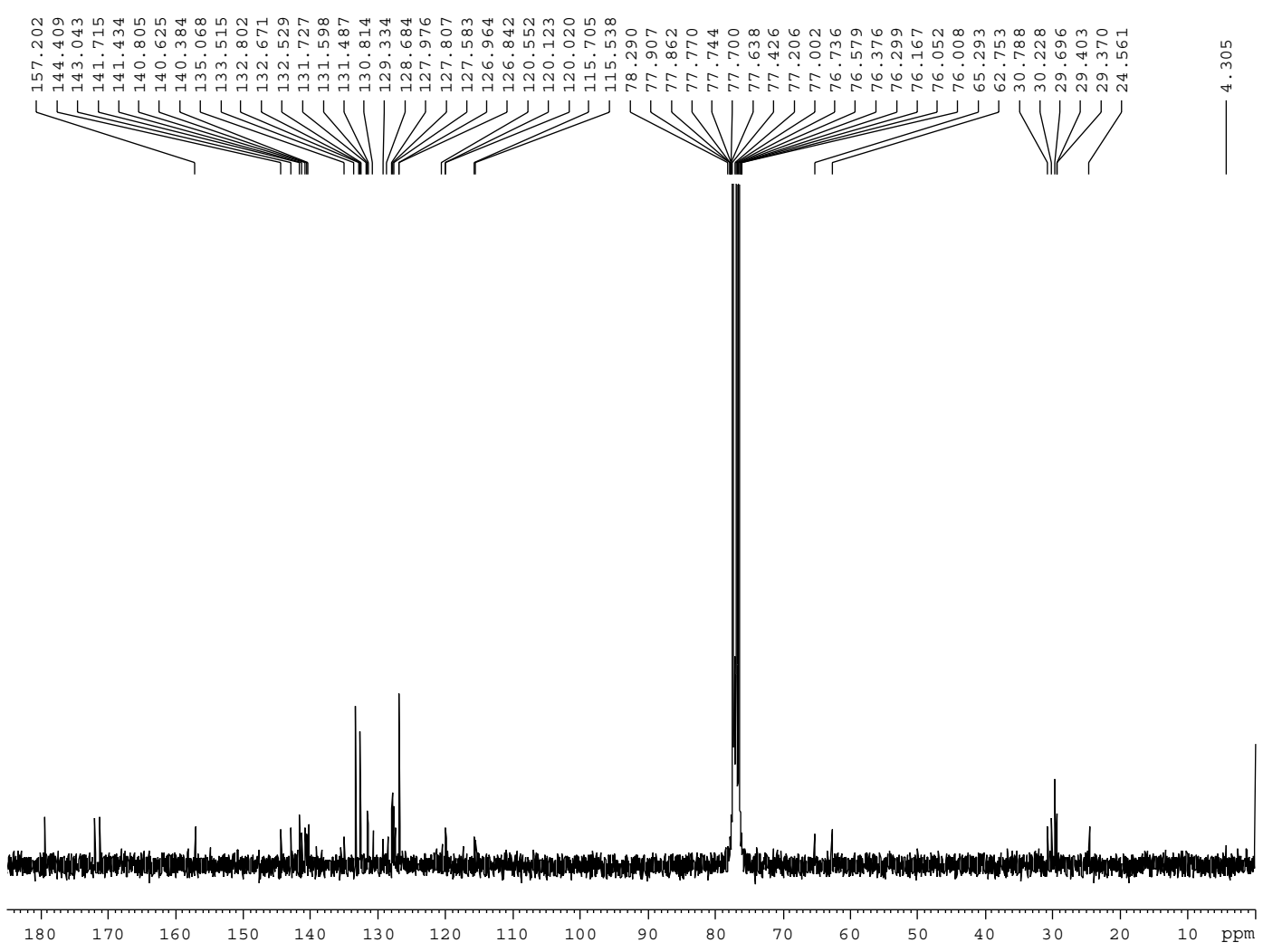

Figure S15. ${ }^{13} \mathrm{C}$ NMR of compound 6 . 


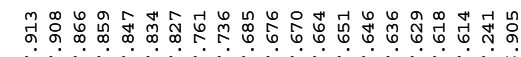

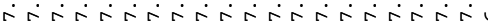

$\longrightarrow$

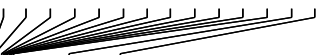

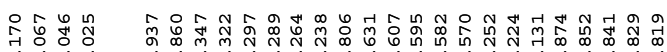

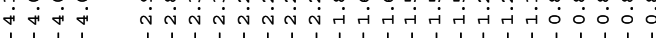

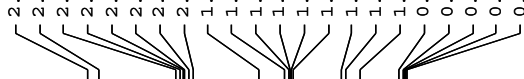
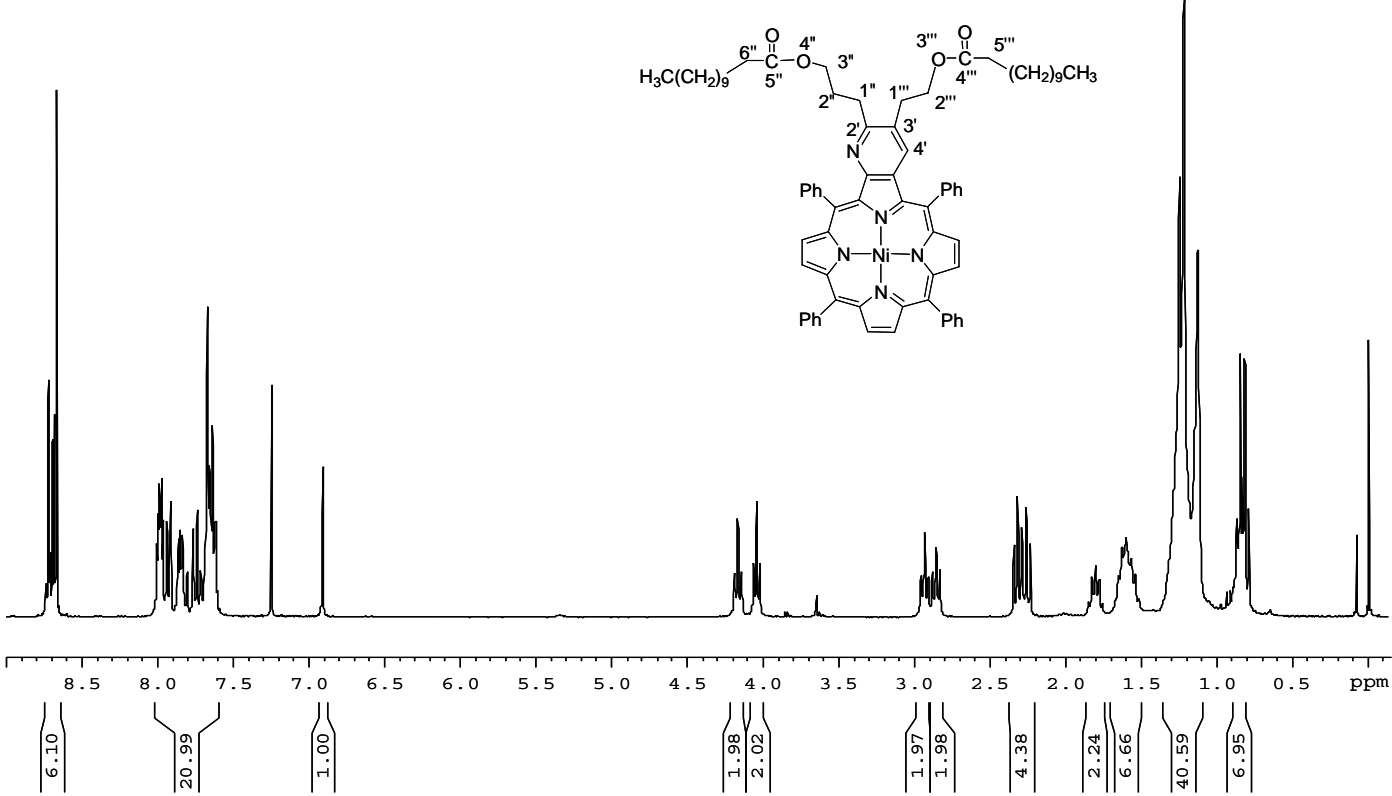

Figure S16. ${ }^{1} \mathrm{H}$ NMR of compound 7.
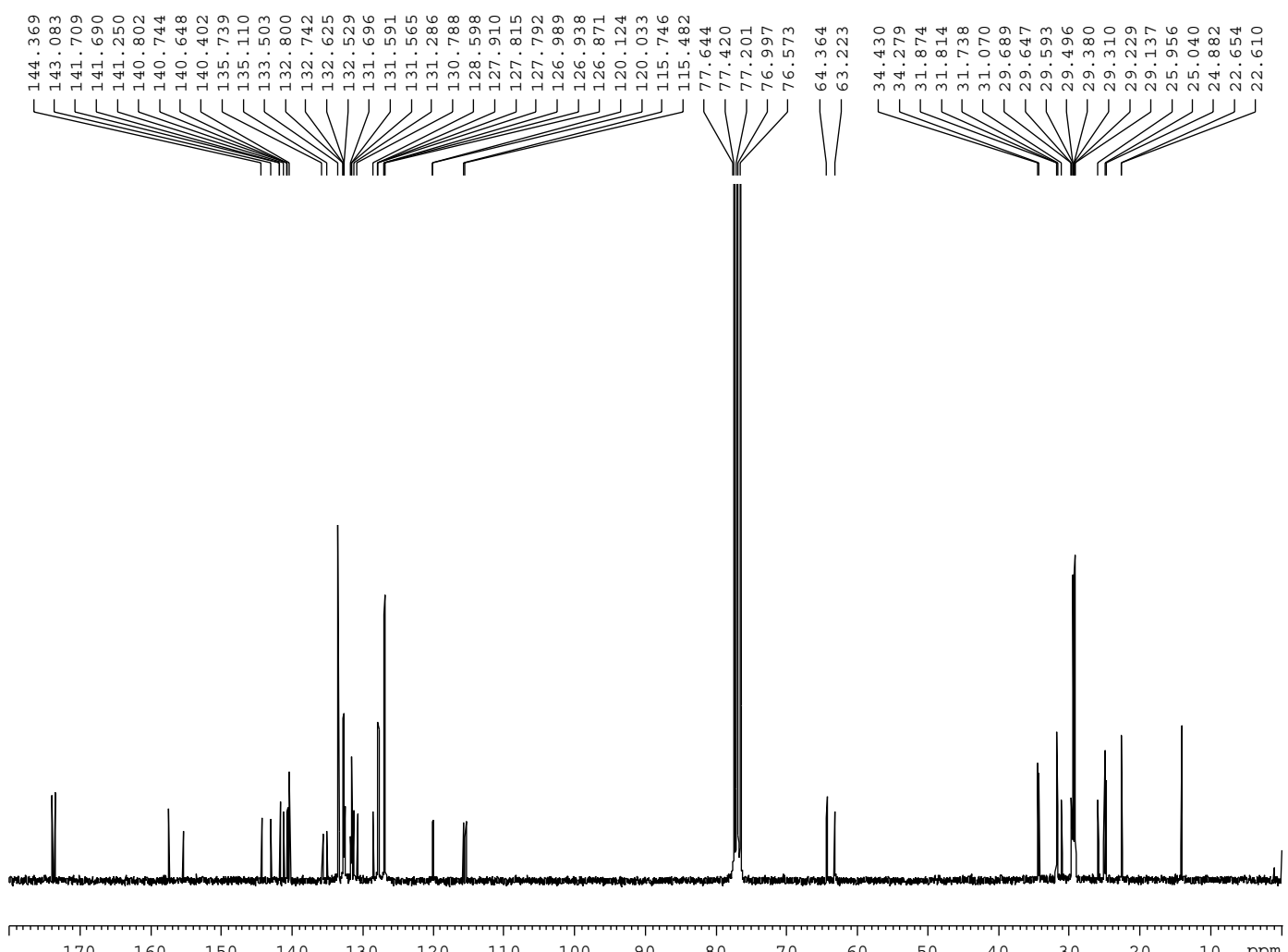

Figure S17. ${ }^{13} \mathrm{C}$ NMR of compound 7. 


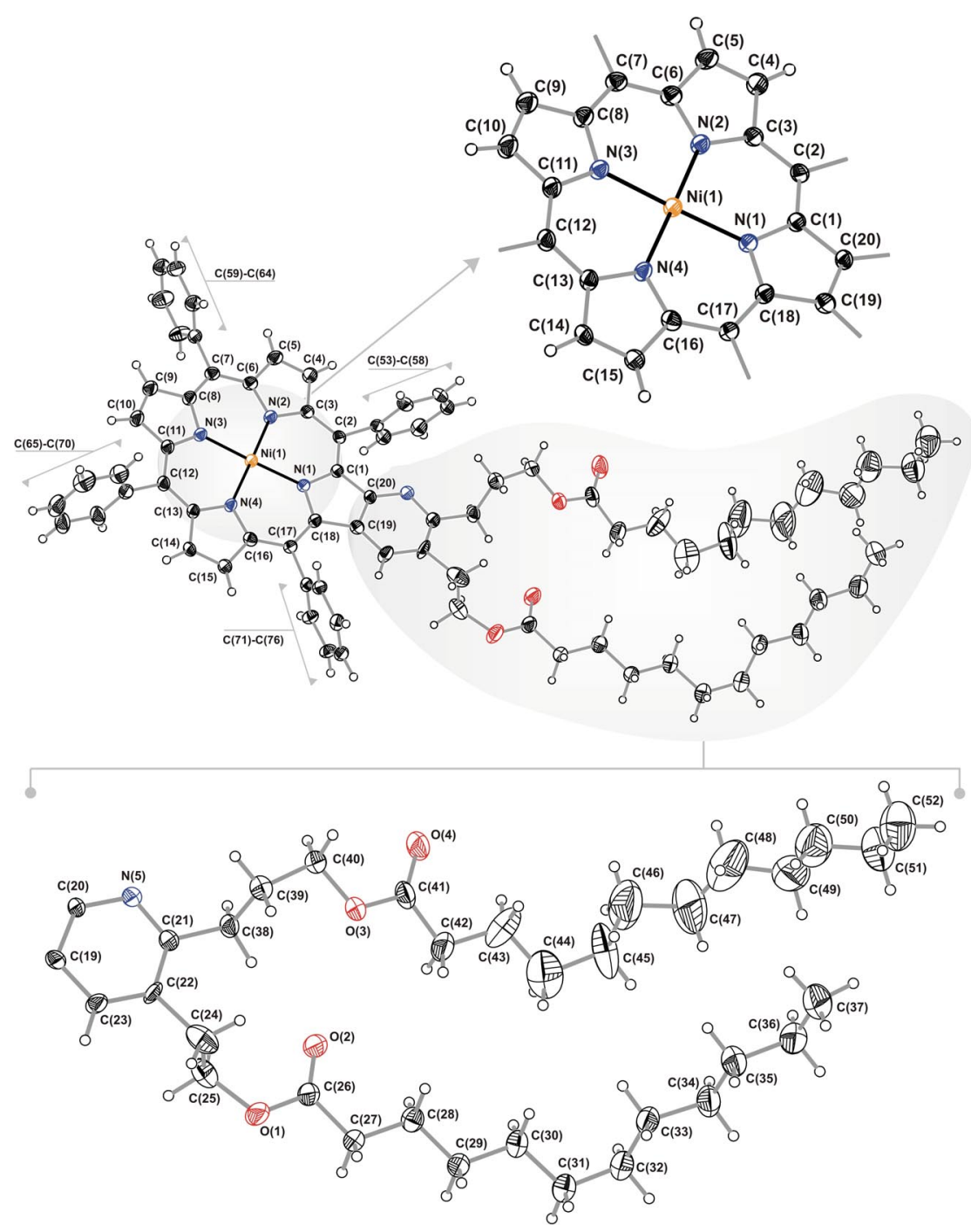

Figure S18. Schematic representation of the molecular unit of porphyrin 7 showing the labelling scheme for all non-hydrogen atoms. Thermal ellipsoids are drawn at the 30\% probability level and hydrogen atoms are represented as small spheres with arbitrary radii. For bond lengths and angles on the $\mathrm{Ni}^{2+}$ coordination environment see Table S2.

Table S1. Crystal and structure refinement data for porphyrin 7.

\begin{tabular}{ll}
\hline & $\mathrm{C}_{76} \mathrm{H}_{83} \mathrm{~N}_{5} \mathrm{NiO}_{4}$ \\
Formula & 1189.18 \\
Formula weight & Triclinic \\
Crystal system & $P \overline{1}$ \\
Space group & $11.2502(3)$ \\
$a / \AA$ & $13.2372(3)$ \\
$b / \AA$ & $23.0320(7)$ \\
$c / \AA$ & $98.059(2)$ \\
$\alpha /^{\mathbf{o}}$ & $94.078(2)$ \\
$\beta / /^{\mathrm{o}}$ & $106.160(2)$ \\
$\gamma / /^{\mathrm{o}}$ & $3239.94(15)$ \\
$\mathrm{Volume}^{\circ} \AA^{3}$ & 2 \\
$Z$ & 1.219
\end{tabular}




\begin{tabular}{|c|c|}
\hline$\mu(\mathrm{Mo}-\mathrm{K} \alpha) / \mathrm{mm}^{-1}$ & 0.847 \\
\hline $\mathrm{F}(000)$ & 1268 \\
\hline Crystal size/mm & $0.18 \times 0.16 \times 0.04$ \\
\hline Crystal type & Red plates \\
\hline$\theta$ range & 1.95 to 54.05 \\
\hline Index ranges & $\begin{array}{l}-11 \leq h \leq 11 \\
-13 \leq k \leq 11 \\
-22 \leq l \leq 23\end{array}$ \\
\hline Reflections collected & 18447 \\
\hline Independent reflections & $7128\left(R_{\text {int }}=0.0422\right)$ \\
\hline Completeness to $\theta=54.05^{\circ}$ & $90.7 \%$ \\
\hline Final $R$ indices $[\mathrm{I}>2 \sigma(\mathrm{I})]^{a, b}$ & $\begin{array}{l}R 1=0.0671 \\
w R 2=0.1813\end{array}$ \\
\hline Final $R$ indices (all data) ${ }^{a, b}$ & $\begin{array}{l}R 1=0.1033 \\
w R 2=0.2096\end{array}$ \\
\hline Weighting scheme ${ }^{c}$ & $\begin{array}{l}m=0.1200 \\
n=3.3912\end{array}$ \\
\hline Largest diff. peak and hole & 0.668 and $-0.555 \mathrm{e}^{-3}$ \\
\hline
\end{tabular}

$$
\begin{aligned}
& { }^{a} R 1=\sum|| F_{o}|-| F_{c}|| / \sum\left|F_{o}\right| ;{ }^{b} w R 2=\sqrt{\sum\left[w\left(F_{o}^{2}-F_{c}^{2}\right)^{2}\right] / \sum\left[w\left(F_{o}^{2}\right)^{2}\right]} \\
& { }^{c} w=1 /\left[\sigma^{2}\left(F_{o}^{2}\right)+(m P)^{2}+n P\right] \text { where } P=\left(F_{o}^{2}+2 F_{c}^{2}\right) / 3
\end{aligned}
$$

Table S2. Selected bond lengths (in $\AA$ ) and angles (in degrees) for the $\mathrm{Ni}^{2+}$ coordination environments present in porphyrin 7 .

\begin{tabular}{llll}
\hline $\mathrm{Ni}(1)-\mathrm{N}(1)$ & $1.969(4)$ & $\mathrm{N}(2)-\mathrm{Ni}(1)-\mathrm{N}(1)$ & $90.05(15)$ \\
$\mathrm{Ni}(1)-\mathrm{N}(2)$ & $1.942(4)$ & $\mathrm{N}(2)-\mathrm{Ni}(1)-\mathrm{N}(3)$ & $90.11(16)$ \\
$\mathrm{Ni}(1)-\mathrm{N}(3)$ & $1.956(4)$ & $\mathrm{N}(3)-\mathrm{Ni}(1)-\mathrm{N}(1)$ & $176.56(16)$ \\
$\mathrm{Ni}(1)-\mathrm{N}(4)$ & $1.938(4)$ & $\mathrm{N}(4)-\mathrm{Ni}(1)-\mathrm{N}(1)$ & $90.80(15)$ \\
& & $\mathrm{N}(4)-\mathrm{Ni}(1)-\mathrm{N}(2)$ & $177.06(17)$ \\
& & $\mathrm{N}(4)-\mathrm{Ni}(1)-\mathrm{N}(3)$ & $89.21(16)$ \\
\hline
\end{tabular}

\section{References}

(1) Giraudeau, A.; Callot, H. J.; Jordan, J.; Ezhar, I.; Gross, M. J. Am. Chem. Soc. 1979, 101, 3857.

(2) Hombrecher, H. K.; Gherdan, V. M.; Ohm, S.; Cavaleiro, J. A. S.; Neves, M. G. P. M. S.; Condesso, M. F. Tetrahedron 1993, 49, 8569.

(3) Kottke, T.; Stalke, D., J. App. Cryst. 1993, 26, 615.

(4) APEX2, Data Collection Software Version 2.1-RC13, Bruker AXS, Delft, The Netherlands 2006.

(5) Cryopad, Remote monitoring and control, Version 1.451, Oxford Cryosystems, Oxford, UnitedKingdom 2006.

(6) SAINT+, Data Integration Engine v. 7.23a ${ }^{\odot}$ 1997-2005, Bruker AXS, Madison, Wisconsin, USA.

(7) Sheldrick, G. M., SADABS v.2.01, Bruker/Siemens Area Detector Absorption Correction Program

1998, Bruker AXS, Madison, Wisconsin, USA. 
(8) Sheldrick, G. M., SHELXS-97, Program for Crystal Structure Solution, University of Göttingen 1997. (9) Sheldrick, G. M., SHELXL-97, Program for Crystal Structure Refinement, University of Göttingen 1997.

(10) Le Page, Y., J. Appl. Crystallogr. 1987, 20, 264.

(11) Le Page, Y., J. Appl. Crystallogr. 1988, 21, 983.

(12) Spek, A. L., J. Appl. Crystallogr. 2003, 36, 7.

(13) Spek, A. L., PLATON, A Multipurpose Crystallographic Tool 2003, Utrecht University, Utrecht, The Netherlands.

(14) Brandenburg, K., DIAMOND, Version 3.1 d. Crystal Impact GbR, Bonn, Germany 2006. 